\title{
Evaluación del empleo generado por el sector medioambiental en la Comunidad de Madrid
}

\author{
Alfredo Cabezas Ares ${ }^{\mathrm{a}}$ \\ Centro de Estudios Superiores Felipe II \\ Julián Moral Carcedo ${ }^{b}$ \\ Universidad Autónoma de Madrid

\section{Raquel Llorente Heras ${ }^{\mathrm{c}}$} \\ Universidad Autónoma de Madrid
}

\begin{abstract}
RESUMEN
En este artículo se pretende delimitar con la mayor exactitud posible el empleo que genera el sector medioambiental en la economía madrileña. Para poder evaluar la evolución del empleo y su tendencia seguimos la metodología propuesta por el informe «Links between the environment, economy and jobs» elaborado por GHK en colaboración con Cambridge Econometrics y el Institute for European Environmental Policy (2007). En este contexto, abordamos cuál es la estructura del empleo medioambiental por activida-

a Alfredo Cabezas Ares. Centro de Estudios Superiores Felipe II Campus de la Universidad Complutense de Madrid en Aranjuez. E-mail: alfredo.cabezas@cesfelipesegundo.com

b Julián Moral Carcedo. Departamento de Análisis Económico. Teoría Económica e Historia Económica. Universidad Autónoma de Madrid. Campus Cantoblanco, 28049 Madrid. E-mail: julian.moral@uam.es

c Raquel Llorente Heras. Departamento de Análisis Económico. Teoría Económica e Historia Económica. Universidad Autónoma de Madrid. Campus Cantoblanco, 28049 Madrid. E-mail: raquel.llorente@uam.es
\end{abstract}

Recibido: febrero de 2010. Aceptado: mayo de 2010. 
des económicas así como su delimitación por puestos de trabajo para finalmente encarar cómo es la distribución geográfica del empleo medioambiental madrileño. Los resultados más relevantes demuestran que el sector medioambiental, en materia de empleo y puestos de trabajo, aúna entre el 3\% y el $4 \%$ de la economía madrileña. Pero lo más importante es el intenso potencial de crecimiento observado de dicho sector.

Palabras claves: medio ambiente, empleo, Madrid.

Códigos JEL: Q52, J21.

\begin{abstract}
In this paper we define as accurately as possible the employment generated by the environmental sector in the region of Madrid. To evaluate the evolution of employment and its trend we follow the methodology developed in the report «Links Between the environment, economy and jobs» elaborated by GHK in collaboration with Cambridge Econometrics and the Institute for European Environmental Policy (2007). In this context, we analyse the structure of environmental employment by economic activities as well as its contribution to the creation of jobs, in order to finally study the geographic distribution of environmental employment inside this region. The main results show that the environmental sector combines between 3\% and 4\% of Madrid's employment and jobs. But the most important finding is the strong rate of growth observed in this sector.
\end{abstract}

Keywords: environment, employment, Madrid.

JEL Classification: Q52, J21.

Abreviaturas utilizadas

OCDE Organización para la Cooperación y el Desarrollo Económico

PNUMA Programa de las Naciones Unidas para el Medio Ambiente

OIT Organización Internacional del Trabajo

OMC Organización Mundial del Comercio

EGS Environmental Goods and Services

ZEVs Zero Emission Vehicles

CNAE Clasificación Nacional de Actividades Económicas

DUAE Directorio de Unidades de Actividad Económica 


\section{INTRODUCCIÓN}

La relación entre el empleo y el medio ambiente se impulsa por una sociedad cada vez más sostenible. A pesar de las reticencias iniciales, a día de hoy se apoya decididamente la creación de «empleos verdes» y se potencia que cada vez haya más ocupados en ámbitos relacionados con el medio ambiente. Desde una perspectiva conceptual, el sector medioambiental es un concepto en evolución, que se entiende más como un conjunto de productores y oferentes de una gama muy variada de bienes y servicios que como un sector propiamente. Haciendo un recorrido por las diversas metodologías utilizadas, vemos que para su delimitación se ha recurrido al CNAE, a las categorías de empleo verde, la definición de «actividades medioambientales» o a «ocupaciones relacionadas con el medio ambiente». Diversas organizaciones internacionales se articulan en base a las «actividades medioambientales», pero su delimitación llega a resultados diferenciados.

La Comisión Europea reconoce al medio ambiente como una fuente de creación de nuevos empleos, tal como queda puesto de manifiesto en su comunicación «Medio Ambiente y Empleo: Hacia una Europa Sostenible» - la política ambiental debería considerarse una gran fuerza impulsora de la inversión y de la construcción de una Europa sostenible, generándose tanto crecimiento como empleo-. En los últimos años, la mayoría de los países occidentales ha experimentado un rápido crecimiento de mercado en el sector medioambiental. Si bien, dicho crecimiento en principio se reducía a los servicios de depuración de aguas residuales y de gestión de residuos, con el tiempo han surgido nuevas oportunidades de mercado y yacimientos de empleo debido al peso cada vez mayor de la legislación ambiental, la innovación tecnológica, así como la preocupación ciudadana por el impacto de las actividades y los productos. Así pues, son cada vez más los estudios que apuntan al medio ambiente como uno de los grandes «yacimientos» de empleo señalando que, a igualdad de inversión, la realizada en este campo produce diez veces más empleo que en la industria o la agricultura ${ }^{1}$.

Ante este hecho, resulta claro que tanto las instituciones nacionales como autonómicas deben tomar un papel activo en materia de medio ambiente. En España, puede constatarse la preocupación por el medio ambiente, así como su integración en la actividad económica, en las numerosas actuaciones legislativas

\footnotetext{
1 «Yacimientos de Empleo en la Gestión Ambiental». Editorial Agrícola Española S.A.
} 
que se han llevado a cabo tanto a nivel nacional como en las diferentes Comunidades Autónomas: La Ley de Prevención y Control Integrado de la Contaminación (IPPC) $)^{2}$ o el Plan de Energías Renovables de España (2005-2010) son un buen ejemplo de ello.

De todo esto se deduce que se trata de un sector con un carácter estratégico, que puede contribuir tanto al crecimiento económico como al bienestar de los ciudadanos. Por ello, resulta importante tratar de cuantificar la importancia que dicho sector tiene sobre el conjunto de la economía, y más concretamente en términos de empleo. Este objetivo, a pesar de su aparente concreción, se enfrenta a la falta de una definición de aceptación general que delimite el sector medioambiental de una manera única. Por otra parte, al margen de la delimitación de las actividades medioambientales aunque ligada a la misma, el problema de la falta de disponibilidad de información a un nivel de desagregación adecuado supone en ocasiones una limitación imposible de salvar. Esta doble problemática ha desembocado en una falta de comparación y de confiabilidad de las cifras estimadas de empleo proporcionadas por distintas fuentes.

En el ámbito específicamente europeo el primer intento de delimitación del sector medioambiental lo constituye la publicación del acuerdo de la Conferencia de Estadísticos de la Unión Europea (1994) acerca de las normas para el establecimiento de un sistema de recogida de datos económicos relativos al medio ambiente (Sistema SERIEE, en sus siglas inglesas) ${ }^{3}$. Dicho sistema quedaba insertado en un marco estadístico previo propuesto por Naciones Unidas para la integración de la contabilidad ambiental y económica, coherente con el Sistema Europeo de Cuentas Económicas Integradas (SEC-95). Este manual contiene la primera propuesta de clasificación de las actividades características ambientales (Single Statistical Classification of Environmental Activities and Facilities, CEPA), así como la metodología para la definición y medida del gasto de protección ambiental realizado por agentes públicos y privados en la economía (Environmental Protection Expenditure Account - EPEA).

La estructura básica de este sistema trata de ser, en la medida de lo posible, compatible con el resto de estadísticas relativas al medio ambiente, actualmente en desarrollo

${ }^{2}$ Legislación/ España: Ley 16/2002, de 1 de julio, de Prevención y Control Integrado de la Contaminación.BOE No 157 02/07/2002

${ }^{3}$ EUROSTAT (1994): Sistema Europeo para la Recogida de Información económica relativa a Medio Ambiente (European System for the Collection on Economic Information on the Environment). Manual Series E, EROSTAT E8. Serie E, Bruselas. 
y perfeccionamiento a escala internacional. Las publicaciones posteriores continúan la línea de perfeccionamiento de las estadísticas ambientales a escala internacional, y entre ellas destaca por su influencia, el Manual elaborado conjuntamente por la OCDE y EUROSTAT ${ }^{4}$, y la propia revisión, publicada por EUROSTAT en 2000, de la Clasificación de Actividades y Gastos de Protección del Medio Ambiente.

En esta línea, las «actividades ambientales» quedarían definidas como aquellas que «producen bienes y servicios para medir, prevenir, limitar o corregir los daños ambientales al agua, el aire y el suelo, así como los problemas relacionados con los desechos, el ruido y los ecosistemas. Las tecnologías, los procesos, los productos y los servicios limpios que reducen el riesgo ambiental y minimizan la contaminación y la utilización de materiales se consideran también parte de la industria de protección del medio ambiente ${ }^{5} »$ Esta definición queda concretada por un listado de actividades medioambientales que quedaría incluidas en la misma y que aparentemente solventaría el problema de obtención de cualquier tipo de información estadística. Lamentablemente la falta de correspondencia con actividades $\mathrm{CNAE}^{6}$, el grado de detalle de las actividades, su carácter multisectorial, en definitiva, la ausencia de información estadística adecuada han determinado que haya sido necesario construir cuentas satélites para cuantificar la aportación de dichas actividades al empleo, o recurrir a encuestas directas a empresas para obtener dicha información, exacerbando el problema de falta de comparación y confianza antes mencionado.

Una de las propuestas que ha tratado de solventar estos problemas es la propuesta de GHK (2007) a petición de la Dirección General de Medio Ambiente de la Comisión Europea. Esta propuesta por un lado amplia la definición de actividades medioambientales, contemplando todos los nexos entre economía y medio ambiente que ampliando la definición de Eurostat (que constituirían las actividades medioambientales «core») con otras actividades en las que el medio ambiente constituye un recurso primario o que dependen de la calidad del medio ambiente. Junto a esta ampliación en la delimitación, GHK estima el peso de las actividades medioambientales en las ramas de actividad CNAE (Clasificación Nacional de Actividades Económicas), permitiendo el empleo de la información estadística recopilada según dicha clasificación en la estimación de

${ }^{4}$ OCDE/EUROSTAT (1999): The environment goods and services industry: manual for data collection and analysis. Paris.

${ }^{5}$ Ídem a la referencia previa.

${ }^{6} \mathrm{La}$ CNAE clasifica a las empresas por la naturaleza de su producción, y no por el tipo de actividades medioambientales que realicen. 
las cifras de actividad del sector medioambiental. Este método, aunque imperfecto, permite obtener una estimación de las cifras de empleo vinculadas a las actividades medioambientales a nivel regional y facilitar su comparación con los datos elaborados siguiendo esta metodología.

Según la metodología propuesta por GHK (2007), las actividades relacionadas con el medio ambiente se pueden agrupar en tres grandes tipos:

1. Actividades en las que el medio ambiente constituye un recurso primario o un factor del procesos productivo: agricultura; explotación forestal; extracción de minerales; producción y distribución de energía eléctrica; captación, depuración y distribución de agua.

Dentro de este grupo se integran tres tipos de actividades relacionadas con el medio ambiente:

a) Actividades basadas en el uso de recursos no renovables, como los combustibles fósiles, metales y minerales.

b) Actividades basadas en el uso de recursos renovables, como la agricultura, madera, industria pesquera, abastecimiento de agua, o productos farmacéuticos naturales basados en la biodiversidad.

c) Producciones ecológicamente sostenibles, como agricultura orgánica, silvicultura sostenible y energía renovable.

2. Actividades relacionadas con la protección y gestión del medio ambiente: reciclaje de residuos; contaminación y control de aguas residuales; gestión ambiental. Dentro de esta definición se incluirían los siguientes grupos de actividades:

a) Cambio tecnológico en los procesos de producción y productos con objeto de reducir el uso de energía así como el nivel de emisiones, incluyendo prácticas de construcción modernas en edificios para reducir el carbono.

b) Actividades de gestión y control de la contaminación, normalmente definido por niveles de gasto realizados en los bienes y servicios producidos. Estas actividades están incluidas dentro de la definición formal de «ecoindustrias». ${ }^{7}$

${ }^{7} \mathrm{La}$ «eco-industrias» son todas las actividades que producen bienes y servicios para medir, evitar, limitar, reducir al mínimo o corregir los daños ambientales causados al agua, al aire y al suelo, así como los problemas relacionados con los residuos, el ruido y los ecosistemas. 
c) Gestión de recursos naturales, medido por niveles de gasto. Estas actividades se encuentran también incluidas dentro de la definición formal de «ecoindustrias».

d) «Productos verdes». Esta definición incluye: productos con «pseudoetiquetas» ${ }^{8}$; edificios con diseño pasivo que resultan eficaces para el ahorro de energía gracias al aislamiento térmico, consiguiendo un acondicionamiento natural; vehículos de emisión cero.

3. Actividades que dependen de la calidad del medio ambiente. Dentro de esta definición se incluyen dos tipos de actividades:

a) Actividades económicas que dependen de la calidad del medio ambiente. La calidad del medio ambiente puede afectar a actividades integradas en diversos sectores:

- Turismo relacionado con el medio ambiente que incluye actividades donde el entorno natural influye en la elección del lugar donde se va a realizar dicha actividad turística. (Ejemplos: visitas a montañas, costas, bosques o lagos; actividades de pesca, senderismo, escalada etc.)

- Actividades recreativas que implican beneficios para la salud.

- Desarrollo de nuevos negocios en áreas donde la calidad del medio ambiente es uno de los factores determinantes en la decisión de inversión.

- Construcción de viviendas con espacios verdes, menos ruido y vistas agradables, como compensación por los elevados precios de las viviendas.

${ }^{8}$ En España sólo existen cuatro sistemas de etiquetado que dan garantías a los consumidores de que compran productos poco dañinos para el medio ambiente: el sistema «green freeze», la etiqueta ecológica comunitaria (más conocida como margarita europea), la etiqueta Aenor-Medio Ambiente y la etiqueta de la Generalitat de Cataluña. 
TABLA 1.-Actividades económicas relacionadas con el medio ambiente

\begin{tabular}{|l|}
\hline Sector \\
\hline A. Actividades en las que el medio ambiente es un recurso natural primario \\
\hline $\begin{array}{l}\text { Agricultura } \\
\text { Agricultura sostenible (cultivos orgánicos) }\end{array}$ \\
\hline $\begin{array}{l}\text { Silvicultura } \\
\text { Explotación sostenible Bosques }\end{array}$ \\
\hline Pesca (excepto recreativa) \\
\hline Minería \\
\hline Electricidad \\
Renovables \\
\hline Agua \\
Distribución de agua \\
\hline B. Actividades relacionadas con la protección y la gestión del medio ambiente \\
\hline B.1 Control y gestión de la contaminación \\
\hline Tratamiento de aguas residuales \\
\hline Control de la contaminación atmosférica \\
\hline Administración Pública \\
\hline Gestión medioambiental privada \\
\hline Recuperación de suelos \\
\hline Control de ruidos \\
\hline I+D medioambiente \\
\hline Monitorización control medio ambiente \\
\hline B.2.-Gestión de recursos \\
\hline Reciclado \\
\hline Protección de la naturaleza \\
\hline C.- Calidad medioambiental \\
\hline Turismo medioambiental (ecoturismo, turismo rural, etc.) \\
\hline
\end{tabular}

Fuente: GHK (2007).

b) Gestión de desastres naturales: incluye servicios de seguros contra desastres naturales (avalanchas, sequías, inundaciones, fuegos, erosión costera, terremotos, tsunamis etc.), además de los gastos en protección medioambiental de 
activos inmuebles y los costes de reconstrucción después de la ocurrencia de desastres naturales.

Las actividades vinculadas con el medio ambiente deben ser entendidas no como un sector o industria en términos estrictos, dado que no son actividades que produzcan bienes o servicios sustitutivos próximos, sino más bien como un conjunto de actividades integradas por empresas y agentes, públicos y privados, heterogéneos productores de una gama muy variada de bienes, servicios y tecnologías para las que el medio ambiente es un recurso natural primario, o la calidad del mismo es fundamental en su actividad, o bien desarrollan actividades relacionadas con la protección y la gestión del medio ambiente.

La cualificación económica, en términos de producción, valor añadido o empleo, de estas actividades es un primer paso en la delimitación del efecto de las actividades medioambientales en la economía. Este paso es posible a partir de la información disponible de los coeficientes de asignación calculados por GHK (2007) para ramas CNAE, los cuales permiten estimar qué proporción del empleo de una rama CNAE puede considerarse como vinculada a la definición de medio ambiente antes expuesta. Dado existe un amplio volumen de información disponible que sigue la clasificación CNAE, es posible obtener estimaciones siguiendo la metodología de GHK (2007) que resultarían inabordables por la ausencia de datos con un nivel de desagregación adecuado. Esta metodología es la seguida en este artículo a la hora de estimar el empleo en medio ambiente en la Comunidad de Madrid y su distribución territorial. Las limitaciones obvias de esta metodología se concentran en la imposibilidad de obtener cifras que resulten comparables con otras estimaciones obtenidas con otras definiciones de medio ambiente y en la imposibilidad de desagregar la información de una rama CNAE en los subtipos de actividades medioambientales que considera GHK. ${ }^{9}$

\section{DATOS DE EMPLEO EN MEDIO AMBIENTE}

Partiendo, por tanto, de la definición de empleo medioambiental anterior, que incluye no sólo aquellos empleos dirigidos a la prevención y tratamiento de la con-

${ }^{9}$ Por ejemplo, resulta imposible discernir el peso de la «agricultura ecológica» dentro del agregado «agricultura, ganadería, caza y actividades de los servicios relacionados con las mismas» o de las «energías renovables» en el agregado "producción y distribución de energía eléctrica». 
taminación, sino también todos los empleos en los que el medio ambiente constituye una pieza clave del proceso productivo, y con el objeto de poder obtener una estimación del empleo medioambiental en la Comunidad de Madrid, se ha partido de un estudio realizado por GHK (2007) en colaboración con Cambridge Econometrics y el Institute for European Environmental Policy. En dicho estudio se recoge un procedimiento para imputar el peso del sector de medio ambiente en función de las distintas ramas de actividad incluidas en la Clasificación Nacional de Actividades Económicas (CNAE-93). En concreto, se parte de la siguiente tabla, para estimar el empleo medioambiental:

Por tanto, según el criterio recogido en la Tabla 2, todas las actividades del sector primario estarían completamente incluidas en la industria medioambiental. En cuanto a la industria manufacturera, su relación con el medio ambiente estaría asociada a las actividades de control de contaminación. Mientras que el sector servicios reflejaría la importancia del medio ambiente en el turismo.

Utilizando los datos de la Tabla 2 junto con los datos de empleo del Directorio de Unidades de Actividad Económica del Instituto de Estadística de la Comunidad de Madrid para el período 2001-2007, en la Tabla 3 estimamos el empleo medioambiental en esta región.

El Directorio de Unidades de Actividad Económica contiene los datos básicos de nombre, localización, empleo, actividad, antigüedad y tipo de sociedad del conjunto de los centros de producción de bienes o servicios en los términos estadísticos normalizados a nivel nacional e internacional (Clasificación Nacional de Actividades Económicas 1993 y Sistema Europeo de Cuentas 1995), o las definiciones normalizadas que en su día sustituyan a las mencionadas, ubicados en la Comunidad de Madrid.

TABLA 2.-Peso del Empleo Medioambiental por Rama de Actividad, CNAE-93

\begin{tabular}{|l|c|c|}
\cline { 2 - 3 } \multicolumn{1}{c|}{} & CNAE - 93 & $\begin{array}{l}\text { Peso del Empleo } \\
\text { Medioambiental }\end{array}$ \\
\hline $\begin{array}{l}\text { Agricultura, ganadería, caza y actividades de los servicios } \\
\text { relacionados con las mismas }\end{array}$ & 01 & $100 \%$ \\
\hline $\begin{array}{l}\text { Silvicultura, explotación forestal y actividades de los } \\
\text { servicios relacionados con las mismas }\end{array}$ & 02 & $100 \%$ \\
\hline $\begin{array}{l}\text { Pesca, acuicultura y actividades de los servicios } \\
\text { relacionados con las mismas }\end{array}$ & 05 & $100 \%$ \\
\hline Extracción y aglomeración de antracita, hulla, lignito y turba & 10 & $100 \%$ \\
\hline
\end{tabular}


TABLA 2 (cont).-Peso del Empleo Medioambiental por Rama de Actividad, CNAE-93

\begin{tabular}{|c|c|c|}
\hline $\begin{array}{l}\text { Extracción de crudos de petróleo y gas natural; actividades } \\
\text { de los servicios relacionados con las explotaciones } \\
\text { petrolíferas y de gas, excepto actividades de prospección }\end{array}$ & 11 & $100 \%$ \\
\hline Extracción de minerales de uranio y torio & 12 & $100 \%$ \\
\hline Extracción de minerales metálicos & 13 & $100 \%$ \\
\hline Extracción de minerales no metálicos ni energéticos & 14 & $100 \%$ \\
\hline Fabricación de maquinaria y material eléctrico & 31 & $2 \%$ \\
\hline $\begin{array}{l}\text { Fabricación de equipo e instrumentos médico-quirúrgicos, } \\
\text { de precisión, óptica y relojería }\end{array}$ & 33 & $2 \%$ \\
\hline Fabricación de muebles; otras industrias manufactureras & 36 & $10 \%$ \\
\hline Reciclaje & 37 & $10 \%$ \\
\hline Producción y distribución de energía eléctrica & 401 & $100 \%$ \\
\hline Captación, depuración y distribución de agua & 41 & $100 \%$ \\
\hline Hostelería & 55 & $7 \%$ \\
\hline $\begin{array}{l}\text { Transporte marítimo, de cabotaje y por vías de navegación } \\
\text { interiores }\end{array}$ & 61 & $25 \%$ \\
\hline Transporte aéreo y espacial & 62 & $25 \%$ \\
\hline Transporte terrestre; transporte por tuberías & 60 & $7 \%$ \\
\hline $\begin{array}{l}\text { Actividades anexas a los transportes; actividades de } \\
\text { agencias de viajes }\end{array}$ & 63 & $7 \%$ \\
\hline Selección y colocación de personal & 745 & $0,7 \%$ \\
\hline Servicios de investigación y seguridad & 746 & $0,7 \%$ \\
\hline Actividades industriales de limpieza & 747 & $0,7 \%$ \\
\hline Actividades empresariales diversas & 748 & $0,7 \%$ \\
\hline $\begin{array}{l}\text { Administración pública, defensa y seguridad social } \\
\text { obligatoria }\end{array}$ & 75 & $1 \%$ \\
\hline Actividades de saneamiento público & 90 & $13 \%$ \\
\hline Actividades asociativas & 91 & $13 \%$ \\
\hline Actividades recreativas, culturales y deportivas & 92 & $13 \%$ \\
\hline Actividades diversas de servicios personales & 93 & $13 \%$ \\
\hline $\begin{array}{l}\text { Actividades de los hogares como empleadores de personal } \\
\text { doméstico }\end{array}$ & 95 & $13 \%$ \\
\hline Organismos extraterritoriales & 99 & $13 \%$ \\
\hline
\end{tabular}

Fuente: GHK (2007). 
El Directorio surge ante la necesidad de disponer de información estadística detallada sobre la totalidad de los sectores económicos que sirva para determinar la evolución de cada uno de ellos, posibilite la elaboración de una contabilidad regional en términos normalizados, y contribuya a la planificación, gestión y evaluación de las políticas sectoriales que desarrolla la Comunidad de Madrid. Al mismo tiempo esta información es utilizada para la realización de encuestas o estudios económicos sectoriales.

El modelo conceptual utilizado para la definición de las unidades que conforman el conjunto del sistema económico ha sido establecido por los Reglamentos 696/93 (Unidades estadísticas de observación y de análisis del sistema de producción en la Comunidad) y 2186/93 (Coordinación comunitaria del desarrollo de los registros de empresas utilizados con fines estadísticos), y por la publicación de EUROSTAT «Ficheros de empresas para fines estadísticos: recomendaciones metodológicas. Vol. 1 Luxemburgo, 1996». En ellos se definen las unidades básicas a utilizar en el análisis funcional de las entidades que forman el sistema de producción; se definen tres unidades: la unidad jurídica, la empresa y la unidad local.

Dado que el objetivo de este trabajo está dirigido a obtener una estimación del empleo medioambiental en la Comunidad de Madrid, es importante tener en cuenta las limitaciones de la información contenida en el Directorio. En primer lugar, se incluyen sólo las unidades locales «con local», es decir, aquellas unidades que ejercen su actividad en un lugar delimitado topográficamente, con un carácter más o menos fijo. Estaríamos, de este modo, considerando un concepto de unidad local relativamente parecido al concepto de «establecimiento». Para aquellas actividades en las que no es posible realizar una adscripción a un lugar determinado, se ha asignado el empleo bien a la sede social, bien al lugar que se considera que es donde se organiza la producción, siendo estas direcciones las que actúan como referentes de la unidad local. No se incluyen, por tanto, las unidades profesionales por cuenta propia, cuya actividad no se ejerce en un lugar relativamente fijo y cuya dirección como lugar donde se organiza la producción es el domicilio particular (que son las que se denominan unidades locales «sin local»). En el ámbito económico, el Directorio recoge las actividades económicas en términos de la Clasificación Nacional de Actividades Económicas 1993, excluyéndose:

a) Los hogares que emplean personal doméstico,

b) Las personas físicas propietarias de bienes inmuebles, que emplean dichos bienes para sus propias necesidades o incluso que los alquilan a terceros y 


\section{c) Los organismos extraterritoriales ${ }^{10}$.}

Por otro lado, el Reglamento comunitario establece como optativo la inclusión de las empresas cuya actividad principal este incluida en las secciones A (Agricultura), B (Pesca) o L (Administración Pública) de la CNAE-93, de ellas el Instituto de Estadística de la Comunidad de Madrid ha decidido excluir las actividades de las secciones A y B. Por este motivo, los datos relativos a ocupados en estas dos actividades han sido extraídos de la Encuesta de Población Activa.

En la distribución de actividades, y por tomar como referencia sólo las unidades locales «con local», los sectores más influidos desde el punto de vista del empleo han sido los relacionados con la Construcción y con las Actividades Anexas al Transporte, sectores con fuerte presencia del minifundismo empresarial y sin localización territorial estable.

También se manifiestan algunas incoherencias en los sectores de actividad pertenecientes a la Administración Pública (sección L de la CNAE) o con fuerte peso de los servicios públicos (secciones $\mathrm{M}$ y $\mathrm{N}$ de la CNAE), pues a pesar de disponer de la totalidad del universo de unidades jurídicas y del volumen de empleo que generan a nivel provincial, no siempre es posible establecer el número y localización de las unidades locales y proceder a las estimaciones de empleo de las mismas.

\section{ESTIMACIÓN DEL EMPLEO MEDIOAMBIENTAL EN LA COMUNIDAD DE MADRID}

La Tabla 3 recoge el número de ocupados en medio ambiente por ramas de actividad según la clasificación CNAE-93, mientras que la Figura 1 muestra la evolución del total de ocupados para el período 2001-2007.

${ }^{10}$ Aunque estas actividades no se encuentran relacionadas con el medio ambiente su exclusión puede implicar cierta sobreestimación del peso global de las actividades medioambientales sobre el total de actividades. 
TABLA 3.- Ocupados en Medio Ambiente por Ramas de Actividad (CNAE-93)

\begin{tabular}{|c|c|c|c|c|c|c|c|}
\hline CNAE-93 & 2001 & 2002 & 2003 & 2004 & 2005 & 2006 & 2007 \\
\hline 01 & 18.400 & 19.825 & 17.175 & 21.650 & 29.150 & 29.950 & 25.900 \\
\hline 10 & 616 & 29 & 29 & 32 & 29 & 50 & 61 \\
\hline 11 & 295 & 344 & 344 & 339 & 456 & 541 & 579 \\
\hline 12 & 254 & 200 & 182 & 182 & 182 & - & - \\
\hline 13 & 65 & 3 & 5 & 5 & 2 & - & - \\
\hline 14 & 1.143 & 1.328 & 1.348 & 1.273 & 1.242 & 1.628 & 1.346 \\
\hline 31 & 281 & 226 & 194 & 201 & 209 & 227 & 241 \\
\hline 33 & 145 & 167 & 207 & 178 & 147 & 119 & 118 \\
\hline 36 & 2.096 & 2.227 & 2.153 & 1.957 & 1.893 & 1.836 & 1.683 \\
\hline 37 & 109 & 128 & 128 & 126 & 132 & 155 & 172 \\
\hline 401 & 6.662 & 7.967 & 8.013 & 13.919 & 14.940 & 18.097 & 11.101 \\
\hline 41 & 2.430 & 2.537 & 2.993 & 3.420 & 3.219 & 3.351 & 3.351 \\
\hline 55 & 8.095 & 8.592 & 8.933 & 9.470 & 9.846 & 10.615 & 12.225 \\
\hline 60 & 3.094 & 3.510 & 3.707 & 4.202 & 4.502 & 4.472 & 5.150 \\
\hline 63 & 1.618 & 1.565 & 1.870 & 1.879 & 2.474 & 2.696 & 2.742 \\
\hline 61 & 166 & 102 & 102 & 102 & 176 & 133 & 146 \\
\hline 62 & 5.519 & 5.414 & 5.453 & 5.293 & 5.647 & 5.993 & 6.061 \\
\hline 745 & 94 & 167 & 185 & 195 & 276 & 309 & 405 \\
\hline 746 & 148 & 175 & 174 & 154 & 205 & 261 & 248 \\
\hline 747 & 289 & 411 & 427 & 466 & 526 & 580 & 660 \\
\hline 748 & 143 & 160 & 195 & 209 & 210 & 243 & 232 \\
\hline 75 & 843 & 902 & 916 & 1.041 & 1.196 & 1.407 & 1.405 \\
\hline 90 & 1.735 & 1.721 & 1.684 & 1.943 & 2.369 & 2.268 & 2.674 \\
\hline 91 & 3.127 & 2.822 & 2.723 & 2.594 & 2.799 & 2.799 & 3.006 \\
\hline 92 & 5.871 & 6.755 & 7.511 & 8.312 & 9.814 & 10.568 & 10.583 \\
\hline 93 & 2.807 & 3.050 & 3.232 & 3.459 & 3.611 & 3.738 & 3.788 \\
\hline Total & 66.044 & 70.326 & 69.883 & 82.600 & 95.253 & 102.035 & 93.876 \\
\hline
\end{tabular}

Fuente: Elaboración propia.

Como se muestra en la Figura 1 el número total de ocupados en actividades medioambientales en la Comunidad de Madrid ha experimentado un importante cre- 
cimiento a lo largo del período 2001-2007, pasando de unos 66.000 empleados en el año 2001 a casi 94.000 en 2007. Si bien, cabe señalar que en el año 2007 se observa un ligero retroceso con respecto al 2006, año en que el empleo medioambiental se situaba en torno a las 102.000 personas. Este resultado pone de manifiesto la importancia del medio ambiente como yacimiento de creación de empleo en la Comunidad de Madrid.

FIgURA 1.-Total Ocupados en el Sector de Medio Ambiente (Comunidad de Madrid)

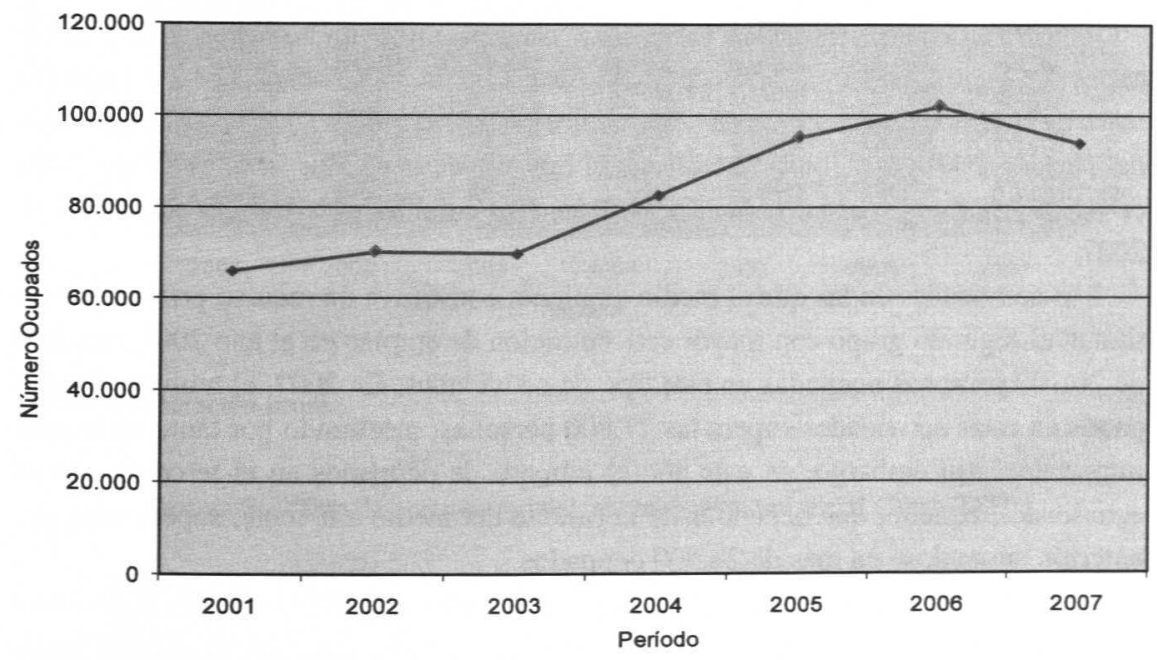

Fuente: Elaboración propia.

Siguiendo la definición de empleo medioambiental propuesta podemos agregar la actividades de la Tabla 2 en los tres grandes grupos de actividades medioambientales: (1) Actividades en las que el medio ambiente constituye un recurso primario; (2) Actividades relacionadas con la protección y gestión del medio ambiente; y (3) Actividades que dependen de la calidad del medio ambiente. La distribución del empleo medioambiental entre estos tres grandes bloques quedaría, por tanto, de la forma recogida en la tabla 4. 
TABLA 4.- Ocupados en medio ambiente por ramas de actividad (datos agregados)

\begin{tabular}{|c|c|c|c|c|c|c|c|}
\cline { 2 - 8 } \multicolumn{1}{c|}{} & $\mathbf{2 0 0 1}$ & $\mathbf{2 0 0 2}$ & $\mathbf{2 0 0 3}$ & $\mathbf{2 0 0 4}$ & $\mathbf{2 0 0 5}$ & $\mathbf{2 0 0 6}$ & $\mathbf{2 0 0 7}$ \\
\hline$(1)$ & 20.773 & 21.729 & 19.083 & 23.481 & 31.061 & 32.169 & 27.886 \\
\hline$(2)$ & 26.560 & 28.864 & 29.764 & 36.864 & 39.258 & 43.188 & 37.435 \\
\hline$(3)$ & 18.711 & 19.734 & 21.036 & 22.255 & 24.934 & 26.678 & 28.556 \\
\hline Total & $\mathbf{6 6 . 0 4 4}$ & $\mathbf{7 0 . 3 2 6}$ & $\mathbf{6 9 . 8 8 3}$ & $\mathbf{8 2 . 6 0 0}$ & $\mathbf{9 5 . 2 5 3}$ & $\mathbf{1 0 2 . 0 3 5}$ & $\mathbf{9 3 . 8 7 6}$ \\
\hline
\end{tabular}

Fuente: Elaboración propia.

Como puede apreciarse en la Tabla 4, en todos los años son las actividades relacionadas con la protección y gestión del medio ambiente las que concentran un mayor número de ocupados en el sector de medio ambiente. Analizando su evolución a lo largo del período 2001-2007, observamos que el número de ocupados dentro de este grupo de actividades ha pasado de unas 26.500 personas en el año 2001 a más de 37.400 en 2007.

Las actividades en las que el medio ambiente constituye un recurso primario constituían el segundo grupo con mayor concentración de empleo en el año 2001, con más de 20.700 personas ocupadas en este tipo de actividades. En 2007, el número de ocupados en estas actividades supera las 27.800 personas, mostrando por tanto un avance importante. Sin embargo, en este año el número de ocupados en el tercer grupo de actividades, aquellas que dependen de la calidad del medio ambiente, supera a la cifra anterior, situándose en más de 28.500 ocupados.

Por otro lado, resulta importante establecer una medida del peso que supone el empleo medioambiental en el total del empleo de la Comunidad de Madrid. Como puede observarse en la Figura 2 el empleo medioambiental supone entre un $3 \%$ y un $4 \%$ del total del empleo en dicha Comunidad. En general, se observa una tendencia ligeramente creciente de la importancia de la industria medioambiental en materia de empleo, a lo largo del período considerado. Si bien, parece producirse un ligero retroceso entre el 2006 y el 2007.

Este aspecto queda reflejado con más detalle en la Figura 3 donde se muestra la tasa de variación interanual en el número de ocupados en medio ambiente. Como puede observarse, son los años 2003-2006 los que experimentan un importante crecimiento relativo del sector en materia de empleo. En concreto, entre los años 2003 y 2004 se registra una tasa de crecimiento interanual en torno al $18 \%$. 
Figura 2.-Evolución Empleo en Medio Ambiente

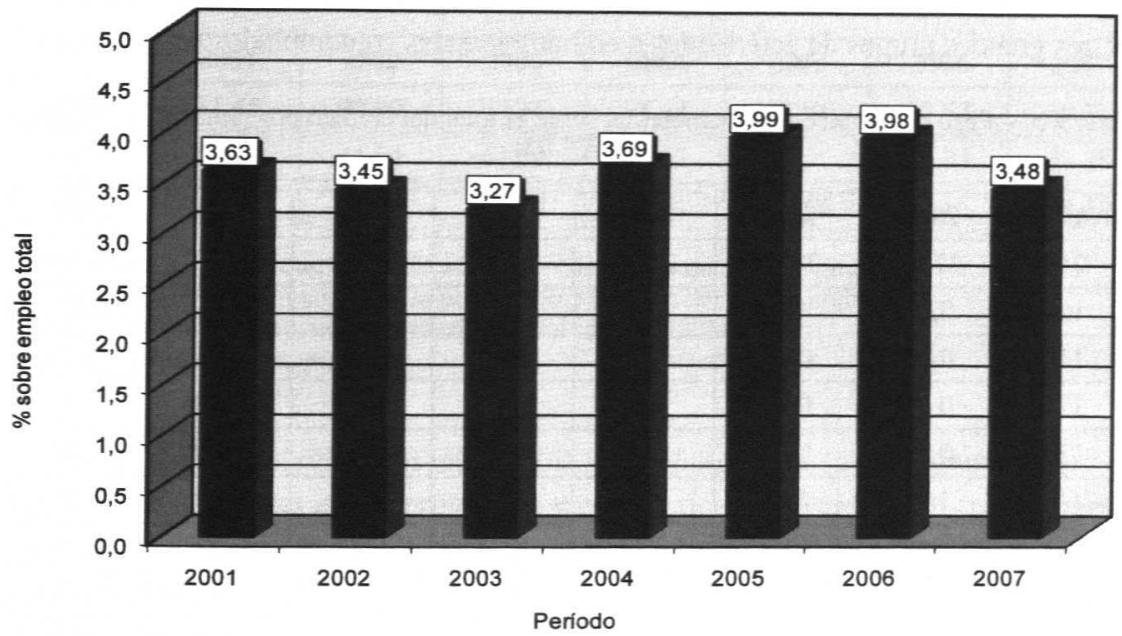

Fuente: Elaboración propia.

FiguRA 3.-Tasa de crecimiento del empleo medioambiental (2001-2007)

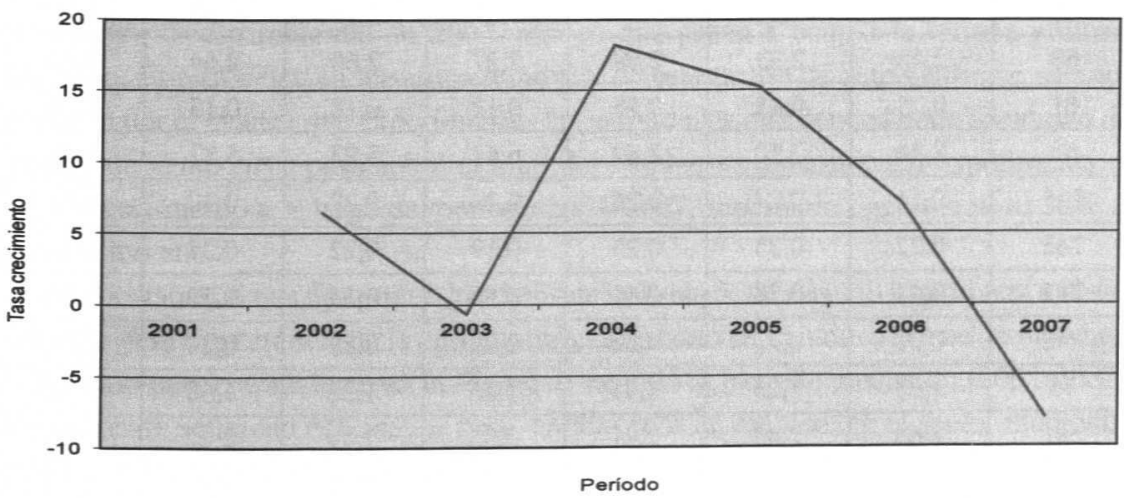

Fuente: Elaboración propia. 
Por otro lado, la Tabla 5 muestra el peso de cada rama de actividad sobre el total del empleo medioambiental, mientras que la Tabla 6 recoge los correspondientes pesos de los tres grandes grupos de actividades medioambientales contemplados en la Tabla 1.

TABLA 5.-Peso del empleo medioambiental sobre el total del empleo por ramas de actividad (CNAE-93)

\begin{tabular}{|c|c|c|c|c|c|c|c|}
\hline CNAE-93 & 2001 & 2002 & 2003 & 2004 & 2005 & 2006 & 2007 \\
\hline 01 & 27,86 & 28,19 & 24,58 & 26,21 & 30,60 & 29,35 & 27,59 \\
\hline 10 & 0,93 & 0,04 & 0,04 & 0,04 & 0,03 & 0,05 & 0,06 \\
\hline 11 & 0,45 & 0,49 & 0,49 & 0,41 & 0,48 & 0,53 & 0,62 \\
\hline 12 & 0,38 & 0,28 & 0,26 & 0,22 & 0,19 & 0,00 & 0,00 \\
\hline 13 & 0,10 & 0,00 & 0,01 & 0,01 & 0,00 & 0,00 & 0,00 \\
\hline 14 & 1,73 & 1,89 & 1,93 & 1,54 & 1,30 & 1,60 & 1,43 \\
\hline 31 & 0,43 & 0,32 & 0,28 & 0,24 & 0,22 & 0,22 & 0,26 \\
\hline 33 & 0,22 & 0,24 & 0,30 & 0,22 & 0,15 & 0,12 & 0,13 \\
\hline 36 & 3,17 & 3,17 & 3,08 & 2,37 & 1,99 & 1,80 & 1,79 \\
\hline 37 & 0,16 & 0,18 & 0,18 & 0,15 & 0,14 & 0,15 & 0,18 \\
\hline 401 & 10,09 & 11,33 & 11,47 & 16,85 & 15,68 & 17,74 & 11,83 \\
\hline 41 & 3,68 & 3,61 & 4,28 & 4,14 & 3,38 & 3,28 & 3,57 \\
\hline 55 & 12,26 & 12,22 & 12,78 & 11,46 & 10,34 & 10,40 & 13,02 \\
\hline 60 & 4,68 & 4,99 & 5,30 & 5,09 & 4,73 & 4,38 & 5,49 \\
\hline 63 & 2,45 & 2,23 & 2,68 & 2,27 & 2,60 & 2,64 & 2,92 \\
\hline 61 & 0,25 & 0,15 & 0,15 & 0,12 & 0,18 & 0,13 & 0,16 \\
\hline 62 & 8,36 & 7,70 & 7,80 & 6,41 & 5,93 & 5,87 & 6,46 \\
\hline 745 & 0,14 & 0,24 & 0,26 & 0,24 & 0,29 & 0,30 & 0,43 \\
\hline 746 & 0,22 & 0,25 & 0,25 & 0,19 & 0,22 & 0,26 & 0,26 \\
\hline 747 & 0,44 & 0,58 & 0,61 & 0,56 & 0,55 & 0,57 & 0,70 \\
\hline 748 & 0,22 & 0,23 & 0,28 & 0,25 & 0,22 & 0,24 & 0,25 \\
\hline 75 & 1,28 & 1,28 & 1,31 & 1,26 & 1,26 & 1,38 & 1,50 \\
\hline 90 & 2,63 & 2,45 & 2,41 & 2,35 & 2,49 & 2,22 & 2,85 \\
\hline 91 & 4,73 & 4,01 & 3,90 & 3,14 & 2,94 & 2,74 & 3,20 \\
\hline 92 & 8,89 & 9,60 & 10,75 & 10,06 & 10,30 & 10,36 & 11,27 \\
\hline 93 & 4,25 & 4,34 & 4,62 & 4,19 & 3,79 & 3,66 & 4,04 \\
\hline
\end{tabular}

Fuente: Elaboración propia. 
TABLA 6.-Distribución o peso del empleo medioambiental sobre el total del empleo por ramas de actividad (datos agregados)

\begin{tabular}{|l|l|l|l|l|l|l|l|}
\cline { 2 - 8 } \multicolumn{1}{c|}{} & $\mathbf{2 0 0 1}$ & $\mathbf{2 0 0 2}$ & $\mathbf{2 0 0 3}$ & $\mathbf{2 0 0 4}$ & $\mathbf{2 0 0 5}$ & $\mathbf{2 0 0 6}$ & $\mathbf{2 0 0 7}$ \\
\hline $\mathbf{( 1 )}$ & 31,45 & 30,90 & 27,31 & 28,43 & 32,61 & 31,53 & 29,71 \\
\hline $\mathbf{( 2 )}$ & 40,22 & 41,04 & 42,59 & 44,63 & 41,21 & 42,33 & 39,88 \\
\hline $\mathbf{( 3 )}$ & $\mathbf{2 8 , 3 3}$ & $\mathbf{2 8 , 0 6}$ & 30,10 & 26,94 & 26,18 & 26,15 & 30,42 \\
\hline
\end{tabular}

(1) Actividades en las que el medio ambiente constituye un recurso primario

(2) Actividades relacionadas con la protección y gestión del medio ambiente

(3) Actividades que dependen de la calidad del medio ambiente

Fuente: Elaboración propia.

$\mathrm{Al}$ igual que observábamos en la Tabla 4, los datos de la Tabla 6 apuntan a las actividades relacionadas con la protección y gestión del medio ambiente, como el grupo con mayor peso en materia de empleo medioambiental en la Comunidad de Madrid. En 2001, alrededor del 40\% del empleo en medio ambiente en la Comunidad de Madrid se concentra en este tipo de actividades, porcentaje que se mantiene en el último año del período analizado. Por otro lado, cabe señalar que este grupo de actividades alcanza su mayor peso, dentro del total de actividades medioambientales, en 2004, año en que el porcentaje superaba el $44 \%$. En cuanto a las actividades en las que el medio ambiente constituye un recurso primario, podemos observar un ligero retroceso en términos relativos, pasando de un $31.45 \%$ en el año 2001 a menos del $30 \%$ del total de empleo medioambiental en 2007 , año en que pasan a ocupar la tercera y última posición. El retroceso, en términos relativos, de este grupo de actividades se ve compensado por el avance que experimentan las actividades que dependen de la calidad del medio ambiente cuyo peso sobre el total del empleo medioambiental experimenta un ligero crecimiento a lo largo del período 2001-2007, superando ligeramente el 30\% en este último año.

No obstante, si nos fijamos en los datos de la Tabla 5, un aspecto importante a destacar es la gran importancia de la agricultura y el turismo en la industria medioambiental. Así, como puede apreciarse en la Figura 4, en todo el período analizado (2001-2007), las ramas de actividad con mayor peso, en términos de empleo, en el sector medioambiental en la Comunidad de Madrid son:

- Agricultura, Ganadería, Caza y Actividades de los Servicios Relacionados con las mismas (Código CNAE-93: 01)

- Hostelería (Código CNAE-93: 55) 
- Producción y Distribución de Energía Eléctrica (Código CNAE-93: 401)

- Actividades Recreativas, Culturales y Deportivas (Código CNAE-93: 92)

En conjunto, estas cuatro ramas de actividad integran entorno al $70 \%$ del empleo medioambiental en la Comunidad de Madrid.

Como se observa en la Tabla 7, en 2001 alrededor de unas 39.000 personas estaban ocupadas en alguna de estas cuatro actividades, ascendiendo dicha cifra a más de 59.000 en el año 2007. En este año, la agricultura constituye, sin duda, la rama de actividad que concentra el mayor número de empleados en medio ambiente, ascendiendo dicha cifra a casi 26.000 personas.

TABLA 7.-Ocupados Ramas de Actividad con mayor peso en medio ambiente

\begin{tabular}{|c|c|c|c|c|c|c|c|}
\hline CNAE-93 & $\mathbf{2 0 0 1}$ & $\mathbf{2 0 0 2}$ & $\mathbf{2 0 0 3}$ & $\mathbf{2 0 0 4}$ & $\mathbf{2 0 0 5}$ & $\mathbf{2 0 0 6}$ & $\mathbf{2 0 0 7}$ \\
\hline 92 & 5.871 & 6.755 & 7.511 & 8.312 & 9.814 & 10.568 & 10.583 \\
\hline 401 & 6.662 & 7.967 & 8.013 & 13.919 & 14.940 & 18.097 & 11.101 \\
\hline 55 & 8.095 & 8.592 & 8.933 & 9.470 & 9.846 & 10.615 & 12.225 \\
\hline 01 & 18.400 & 19.825 & 17.175 & 21.650 & 29.150 & 29.950 & 25.900 \\
\hline Total & $\mathbf{3 9 . 0 2 8}$ & $\mathbf{4 3 . 1 3 9}$ & $\mathbf{4 1 . 6 3 1}$ & $\mathbf{5 3 . 3 5 1}$ & $\mathbf{6 3 . 7 5 1}$ & $\mathbf{6 9 . 2 3 0}$ & $\mathbf{5 9 . 8 0 9}$ \\
\hline
\end{tabular}

Fuente: Elaboración propia.

Por tanto, y a raíz de los datos recogidos en la tabla anterior, el primer aspecto a señalar es que son las actividades del sector agrario las que representan un mayor peso en el conjunto del empleo medioambiental, llegando en algunos casos a superar el $30 \%$ del total. La Hostelería es la segunda rama con mayor peso en empleo medioambiental en los primeros años del período analizado, mientras que la Producción y Distribución de Energía Eléctrica ocupa el tercer lugar. Si bien, cabe señalar el importante crecimiento que experimenta esta última en el año 2004, pasando de suponer alrededor de un 15\% del total en el año 2003 a casi un $23 \%$ en el año 2004, convirtiéndose en la segunda rama de actividad con mayor representatividad en el conjunto del sector medioambiental. No obstante, este avance parece ser temporal, de manera que en los próximos años se vuelven a porcentajes más bajos, y en 2007 la Hostelería vuelve a convertirse, de nuevo, en la rama con el segundo mayor peso en el sector medioambiental de la Comunidad de Madrid. 
FiguRA 4..--Ramas de Actividad con mayor peso en medio ambiente

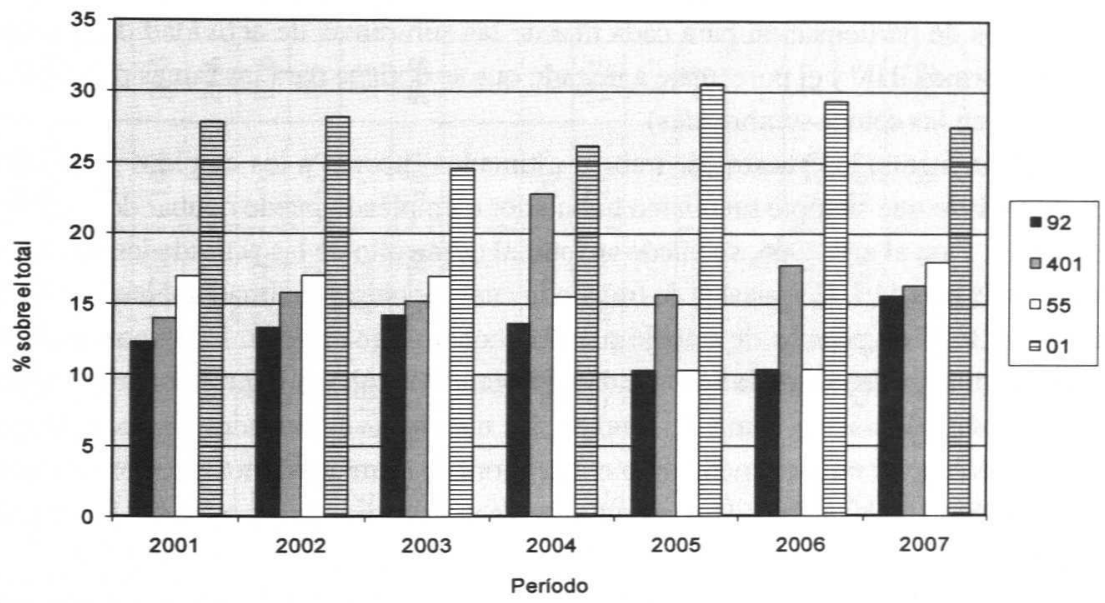

Fuente: Elaboración propia.

\section{AMPliaCión HACIA LOS PUESTOS DE TRABAJO}

Siguiendo con los encadenamientos establecidos anteriormente, un ejercicio paralelo a las estimaciones previas sobre el empleo consiste en establecer cuál es la importancia del sector medioambiental en términos de los puestos de trabajo asociados a dichos empleos. Esta perspectiva amplía y refuerza los cálculos anteriores al acudir a una nueva fuente de datos: las Cuentas Económicas de la Comunidad de Madrid que albergan datos sobre el empleo y la producción para cada una de las actividades productivas madrileñas ${ }^{11}$. No obstante, dichos datos se encuentran agregados por grandes ramas ofreciendo, además, una clasificación propia de las actividades productivas y, por ello, no comparable directamente con la utilizada por GHK. Ello, impide el correcto uso de los porcentajes de participación dado que no se alcanza el suficiente grado de detalle para el cual son definidos provocando ciertas sobreestimaciones ${ }^{12}$. A pesar

${ }^{11}$ En el momento de elaboración de este artículo los datos más recientes de carácter adelantado se constituyen para el año 2006.

${ }^{12}$ Asimismo, la escasa definición de actividades impide distinguir el sector medioambiental de forma más estricta; por lo cual se aúna la totalidad de puestos de trabajo sin distinguir por la clasificación utilizada en el apartado anterior en torno a tres categorías básicas. 
de este inconveniente, en la tabla anexa se recoge los encadenamientos asumidos o porcentajes de participación para cada una de las sub-ramas de actividad de acuerdo con el informe GHK y el porcentaje agregado que se obtiene para las ramas principales (recogido en las celdas sombreadas).

Evidentemente, los puestos de trabajo estimados superan a los empleos medioambientales dado que siempre un mismo trabajador o empleado puede ocupar dos puestos de trabajo. Para el año 2006, se puede atribuir al desarrollo de las actividades anexas al medio ambiente 119.983 puestos de trabajo lo cual supone aproximadamente el 3,7 por ciento del total de puestos de trabajo para la economía madrileña. La importancia de estas actividades dentro de la Comunidad de Madrid resulta similar tanto en términos de los puestos de trabajo como del empleo. De nuevo, las actividades productivas que acaparan un mayor empleo medioambiental, ahora en términos de los puestos de trabajo, en Madrid son las ramas de Agricultura y ganadería, Energía y minería, Hostelería y Otros servicios.

El crecimiento de los puestos de trabajo anexos al medioambiente se estima para el período 2002-2006 en torno al 16 por ciento; se trata por tanto de actividades en desarrollo. Las actividades que durante este período han visto incrementar sus puestos de trabajo medioambientales en mayor medida son las ramas de Servicios a empresas (con un crecimiento del 24,6 por ciento); Transportes y Comunicaciones (con un crecimiento del 21,0 por ciento) y Hostelería (con un crecimiento del 20,7 por ciento). 


\begin{tabular}{|c|c|c|c|c|c|c|c|c|}
\hline 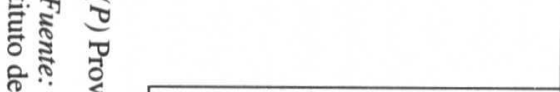 & $\begin{array}{c}\text { Porcentajes de } \\
\text { participación }\end{array}$ & 2002 & 2003 & 2004 & $2005(P)$ & $2006(A)$ & $\begin{array}{c}\text { Crec. } \\
2002-6\end{array}$ & $\begin{array}{c}\% \\
2006\end{array}$ \\
\hline Agricultura y ganadería & 100 & 18.667 & 18.951 & 20.721 & 21.758 & 22.280 & 19,4 & 18,6 \\
\hline 1. Agricultura y ganadería & 100 & 18.667 & 18.951 & 20.721 & 21.758 & 22.280 & 19,4 & 18,6 \\
\hline Industria & 7,6 & 24.110 & 24.404 & 24.941 & 25.390 & 25.934 & 7,6 & 21,6 \\
\hline 2. Energía y minería & 100 & 19.327 & 19.496 & 20.157 & 20.557 & 21.091 & 9,1 & 17,6 \\
\hline 6. Material eléctrico y electrónico & 2 & 697 & 680 & 669 & 673 & 659 & $-5,5$ & 0,5 \\
\hline 13. Otras manufactureras & 10 & 4.086 & 4.229 & 4.116 & 4.160 & 4.184 & 2,4 & 3,5 \\
\hline Servicios & 2,9 & 60.681 & 63.792 & 65.968 & 68.729 & 71.770 & 18,3 & 59,8 \\
\hline $\begin{array}{l}\text { Comercio, hostelería, transporte y } \\
\text { comunicaciones }\end{array}$ & & & & & & & & \\
\hline 17. Hostelería & 7 & 9.991 & 10.371 & 10.823 & 11.495 & 12.056 & 20,7 & 10 \\
\hline 18. Transportes y comunicaciones & 8 & 17.143 & 17.852 & 18.613 & 19.628 & 20.788 & 21,3 & 17,3 \\
\hline \multicolumn{9}{|c|}{ Inmobiliarias y alquileres, servicios a empresas y financieros } \\
\hline 20. Servicios a empresas & 0,7 & 2.649 & 2.738 & 2.927 & 3.056 & 3.300 & 24,6 & 2,8 \\
\hline \multicolumn{9}{|l|}{ Otros servicios } \\
\hline 26. Otros servicios & 13 & 29.096 & 31.028 & 31.740 & 32.591 & 33.635 & 15,6 & 28 \\
\hline 27. Administraciones públicas & 1 & 1.801 & 1.803 & 1.865 & 1.959 & 1.991 & 10,5 & 1,7 \\
\hline $\begin{array}{l}\text { Total de actividades } \\
\text { medioambientales }\end{array}$ & 3,8 & 103.457 & 107.147 & 111.629 & 115.876 & 119.983 & 16 & 100 \\
\hline $\begin{array}{l}\text { Crec. anual de las act. } \\
\text { medioambientales }\end{array}$ & & & 3,6 & 4,2 & 3,8 & 3,5 & & \\
\hline $\begin{array}{l}\text { Peso de las act. } \\
\text { Medioambientales }\end{array}$ & & 3,8 & 3,8 & 3,8 & 3,8 & 3,7 & & \\
\hline
\end{tabular}




\section{DiSTRIBUCIÓN GEOGRÁFICA DEL EMPLEO EN LAS ACTIVIDADES

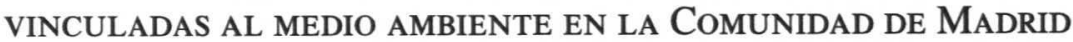

Como información complementaria a la estimación del empleo en actividades medioambientales, en este apartado se aborda el estudio de la localización geográfica del mismo en el marco de la Comunidad de Madrid. En este estudio se analiza la distribución del empleo en las distintas áreas de la Comunidad de Madrid, las cuales quedan conformadas por el Municipio de Madrid (Almendra Central, Periferia Noroeste, Periferia Este y Periferia Sur), corona metropolitana (Norte, Este, Sur y Oeste) y los municipios no metropolitanos. Un mayor detalle de la configuración de estas áreas puede consultarse en el anexo.

\subsection{Delimitación actividades vinculadas al medio ambiente y descripción de los datos}

La información utilizada en este estudio procede del Directorio de Unidades de Actividad Económica (DUAE) de la Comunidad de Madrid y se refieren al año 2007, último período para el que existe información en la fecha de realización de este informe. Dadas las características de esta fuente de datos es preciso tener en cuenta que no se computa el empleo de las actividades del sector primario (agricultura, ganadería y pesca), y por tanto existe de partida una subestimación del empleo medioambiental. Para más información, puede consultarse el detalle de la metodología seguida por el DUAE.

A la hora de delimitar el sector medioambiental se ha partido de la definición proporcionada por GHK. En la tabla siguiente se detallan los sectores y actividades relacionadas con el medio ambiente según la clasificación CNAE a 2 dígitos, así como los porcentajes de asignación a actividades medioambientales de las cifras sectoriales. Estos porcentajes tratan de reflejar qué proporción de la actividad del sector agregado corresponde más directamente con las actividades vinculadas con el medio ambiente, cifras que proceden del estudio realizado por GHK (2007). Debido a la agregación sectorial considerada (CNAE-2 dígitos) la aplicación directa de los coeficientes de asignación generaría divergencias respecto a las cifras obtenidas a partir de la CNAE-4 dígitos. Para evitar estas diferencias se han recalculado los porcentajes de asignación de las actividades que presentaban mayores desviaciones («Producción y distribución de energía eléctrica, gas, vapor y agua caliente» $\mathrm{y}$ "Otras actividades empresariales.») para que el resultado obtenido por la CNAE-2 dígitos coincidiese con el resultado de la CNAE-4 dígitos. En la 
tabla siguiente se indican los porcentajes originales y entre paréntesis los porcentajes resultantes de la reponderación de los sectores con mayores divergencias.

TABLA 9.-Actividades económicas relacionadas con el medio ambiente, CNAE-93

(2 dígitos) y porcentajes de asignación

\begin{tabular}{|c|c|c|}
\hline CNAE & Actividad & $\begin{array}{l}\text { Porcentaje de } \\
\text { Asignación }\end{array}$ \\
\hline 10 & Extracción y aglomeración de antracita, hulla, lignito y turba. & 100 \\
\hline 11 & $\begin{array}{l}\text { Extracción de crudos de petróleo y gas natural. Actividades de los } \\
\text { servicios relacionados con las explotaciones petrolíferas y de gas, excepto } \\
\text { actividades de prospección. }\end{array}$ & 100 \\
\hline 12 & Extracción de minerales de uranio y torio. & 100 \\
\hline 13 & Extracción de minerales metálicos. & 100 \\
\hline 14 & Extracción de minerales no metálicos ni energéticos. & 100 \\
\hline 31 & Fabricación de maquinaria y material eléctrico. & 2 \\
\hline 33 & $\begin{array}{l}\text { Fabricación de equipo e instrumentos médico-quirúrgicos, de precisión, } \\
\text { óptica y relojería. }\end{array}$ & 2 \\
\hline 36 & Fabricación de muebles. Otras industrias manufactureras. & 10 \\
\hline 37 & Reciclaje. & 10 \\
\hline 40 & Producción y distribución de energía eléctrica, gas, vapor y agua caliente. & $100(85,5)$ \\
\hline 41 & Captación, depuración y distribución de agua. & 100 \\
\hline 55 & Hostelería. & 7 \\
\hline 60 & Transporte terrestre. Transporte por tubería. & 7 \\
\hline 61 & Transporte marítimo y por vías de navegación interiores & 25 \\
\hline 62 & Transporte aéreo y espacial. & 25 \\
\hline 63 & Actividades anexas a los transportes; actividades de agencias de viajes. & 7 \\
\hline 74 & Otras actividades empresariales. & $0,7(0,37)$ \\
\hline 75 & Administración pública, defensa y seguridad social obligatoria. & 1 \\
\hline 90 & Actividades de saneamiento público. & 13 \\
\hline 91 & Actividades asociativas. & 13 \\
\hline 92 & Actividades recreativas, culturales y deportivas. & 13 \\
\hline 93 & Actividades diversas de servicios personales. & 13 \\
\hline
\end{tabular}

Fuente: Elaboración propia y GHK (2007).

A la hora de interpretar los resultados debe tenerse en cuenta que la información disponible no permite identificar, dentro de un sector, la ubicación concreta de las unidades económicas que realizan una actividad vinculada al medioambiente. Esta limitación supone que la asignación realizada replica la distribución geográfica del total sectorial, de modo que 
las diferencias existentes quedan enmascaradas. Por ejemplo, en el caso de las actividades de «hostelería», el porcentaje de asignación se aplica al total del sector y su distribución geográfica aparece concentrada en el municipio de Madrid. Sin embargo cabe esperar que las actividades de hostelería vinculadas al medio ambiente (eco turismo fundamentalmente) se encuentren más concentradas en los municipios no pertenecientes al área metropolitana.

\subsection{Aspectos geográficos de las actividades medioambientales}

A grandes rasgos las actividades medioambientales ${ }^{13}$ presentan una distribución geográfica del empleo similar a la del conjunto de las actividades económicas de la Comunidad de Madrid. Esta distribución se caracteriza por una notable concentración del empleo en el municipio de Madrid, donde se ubicaría más de la mitad del total de empleo regional, y en la corona metropolitana. En el caso del empleo total, en Madrid se localizaría el $62,3 \%$ del empleo y en la corona el 29,3\% del empleo. Respecto a las actividades medioambientales, el municipio de Madrid concentraría el 69,3\% del empleo del sector, y la corona metropolitana el $21,9 \%$ del empleo.

Comparando la estructura del empleo del total de la economía regional y del sector medioambiental puede apreciarse en este último caso una tendencia a la concentración en el municipio de Madrid y en el área no metropolitana. Así, el porcentaje del empleo en Madrid municipio se eleva al $69,3 \%$ frente al $62 \%$ en el caso del total de empleo. En el caso de los municipios no metropolitanos dichos porcentajes se elevan al 8,8\% y $8,4 \%$ respectivamente.

FIGURA 5.-Distribución geográfica del empleo sector medioambiental.2007
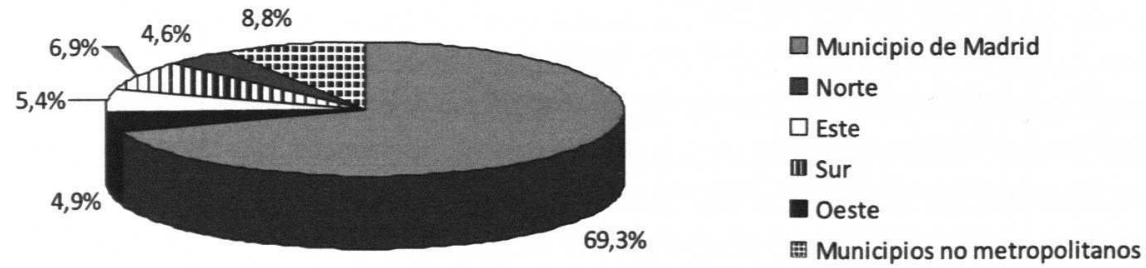

Fuente: Elaboración propia a partir de datos del Directorio de Unidades de Actividad Económica. Instituto Estadística Comunidad de Madrid.

${ }^{13}$ Excluidas las actividades agrícolas, ganaderas y pesqueras. 
FiguRA 6.-Distribución geográfica del empleo Total sectores. 2007

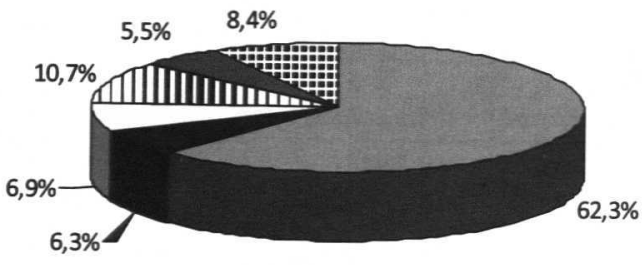

\author{
$\square$ Municipio de Madrid \\ Norte \\ $\square$ Este \\ m Sur \\ Oeste \\ \# Municipios no metropolitanos
}

Fuente: Elaboración propia a partir de datos del Directorio de Unidades de Actividad Económica. Instituto Estadística Comunidad de Madrid.

En las tablas siguientes se presentan los datos detallados de empleo en actividades medioambientales en las distintas áreas geográficas, así como la distribución por sectores. Asimismo se incluyen los valores del índice de especialización geográfica calculado para analizar las diferencias en la estructura geográfica del empleo y que se define según la siguiente expresión:

$$
I E_{j}=\frac{E M j / \sum_{j} E M j}{E j / \sum_{j} E j} \times 100,
$$

Donde,

- EMj, empleo medioambiental en el área geográfica j.

- $\sum_{j} E M j$ empleo total medioambiental en la totalidad de áreas.

- Ej, empleo total en el área geográfica j.

- $\sum_{j} E j$ empleo total en la totalidad de áreas, es decir, en la Comunidad de Madrid.

Si el índice de especialización es mayor de 100 indicaría una mayor concentración del empleo medioambiental en esa área, mientras que un valor inferior a 100 indicaría el caso opuesto, es decir, una presencia del empleo medioambiental inferior a la que presenta el empleo total y por lo tanto indicaría una menor especialización de esa área geográfica en actividades medioambientales. 
TABLA 10.-Distribución del empleo en actividades vinculadas al medio ambiente. Total 2007

\begin{tabular}{|c|c|c|c|c|c|c|c|}
\hline \multicolumn{3}{|c|}{ 总高总 } & ஓ్ & $\frac{\infty}{\vec{d}}$ & \multirow{14}{*}{ 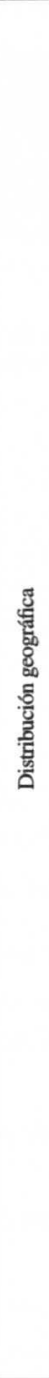 } & $\begin{array}{l}\infty \\
\infty \\
\infty\end{array}$ & $\frac{\stackrel{8}{+}}{\infty}$ \\
\hline \multirow{11}{*}{ 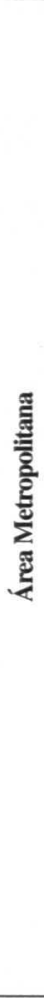 } & \multirow{5}{*}{ 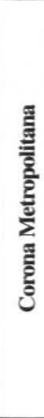 } & 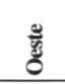 & $\frac{\bar{n}}{m}$ & 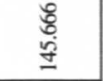 & & \begin{tabular}{l}
80 \\
\hdashline \\
-
\end{tabular} & $\begin{array}{l}g^{\circ} \\
v^{2}\end{array}$ \\
\hline & & 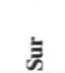 & $\begin{array}{l}\stackrel{0}{0} \\
+ \\
+\end{array}$ & $\begin{array}{l}\hat{\sigma} \\
\text { w. } \\
\text { d. }\end{array}$ & & ఏి & $\frac{8}{\stackrel{8}{0}}$ \\
\hline & & 童 & 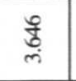 & $\begin{array}{l}\frac{m}{N} \\
\infty \\
\infty\end{array}$ & & $\frac{\stackrel{8}{v}}{n}$ & gे \\
\hline & & $\frac{\pi}{\frac{5}{2}}$ & $\underset{m}{m}$ & $\begin{array}{l}\bar{x} \\
0 \\
0 \\
\underline{0}\end{array}$ & & $\stackrel{\circ}{\circ}$ & $\mathrm{j}_{6}^{\circ}$ \\
\hline & & 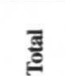 & $\begin{array}{l}\infty \\
\infty \\
\dot{x} \\
\dot{\Psi}\end{array}$ & 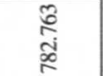 & & $\frac{\Delta}{2}$ & స్ \\
\hline & \multirow{5}{*}{ 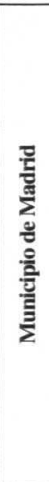 } & 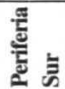 & $\underset{\substack{+\dot{\sim}}}{+}$ & \begin{tabular}{l} 
vै \\
\multirow{2}{*}{} \\
$\vdots$
\end{tabular} & & స్ำ & $\stackrel{8}{2}$ \\
\hline & & 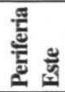 & 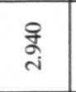 & $\begin{array}{l}\text { הี } \\
\text { ज्ञ }\end{array}$ & & $\stackrel{8}{\stackrel{7}{7}}$ & 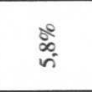 \\
\hline & & 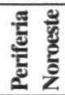 & 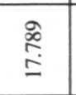 & 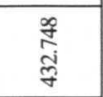 & & ڤ్ & 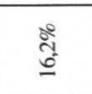 \\
\hline & & 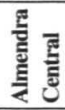 & $\begin{array}{l}\stackrel{n}{\hat{~}} \\
\text { 今े }\end{array}$ & $\begin{array}{l}\underset{\infty}{\infty} \\
\stackrel{8}{0} \\
\infty\end{array}$ & & 总 & స్ \\
\hline & & 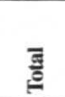 & $\begin{array}{l}0 \\
\stackrel{5}{+} \\
\dot{f}\end{array}$ & 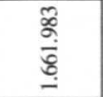 & & $\frac{i^{\circ}}{a^{2}}$ & तु \\
\hline & 梠 & & 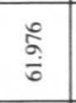 & 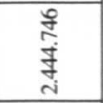 & & $\frac{\tilde{c}}{\sigma}$ & $\frac{80}{a}$ \\
\hline 롱 & & & $\frac{5}{5}$ & 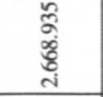 & & $\frac{8}{8}$ & $\begin{array}{l}\stackrel{8}{\circ} \\
\underline{8}\end{array}$ \\
\hline & & & 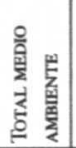 & 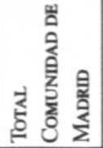 & & 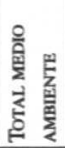 & 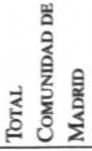 \\
\hline
\end{tabular}

Fuente: Elaboración propia a partir de datos del Directorio de Unidades de Actividad Económica. Instituto Estadística Comunidad de Madrid.

Obsérvese cómo al acudir a diferentes fuentes se ofrece una cuantificación muy detallada del sector medioambiental pero se rompe la comparación entre las mismas debido a la utilización de diferentes metodologías como al diferente período para la disponibilidad de datos. 
EVALUACIÓN DEL EMPLEO GENERADO POR EL SECTOR MEDIOAMBIENTAL

TABLA 11.-Participación del empleo en actividades vinculadas al medio ambiente respecto del empleo total de cada área. Total 2007

\begin{tabular}{|c|c|c|c|c|c|c|}
\hline & \multicolumn{2}{|c|}{ 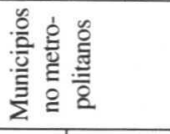 } & 各 & $\frac{\infty}{\vec{d}}$ & $\begin{array}{l}2^{\circ} \\
\hat{i}^{0}\end{array}$ & 守 \\
\hline \multirow{11}{*}{ 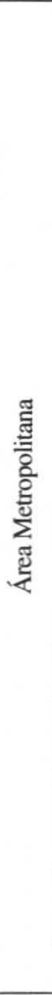 } & \multirow{5}{*}{ 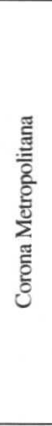 } & $\tilde{8}$ & $\frac{\bar{n}}{m}$ & $\begin{array}{l}\text { 莒 } \\
\text { 守 }\end{array}$ & $\frac{d 0}{i}$ & $\frac{\partial}{\dot{x}}$ \\
\hline & & 点 & 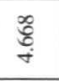 & 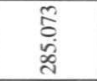 & $\stackrel{0^{\circ}}{-}$ & $\frac{7}{8}$ \\
\hline & & $\frac{\mathscr{y}}{4}$ & 苦 & $\begin{array}{l}\stackrel{m}{d} \\
\stackrel{m}{0} \\
\underline{0}\end{array}$ & $\stackrel{8}{2}$ & $\bar{\infty}$ \\
\hline & & हूँ & $\stackrel{\leftrightarrow}{m}$ & $\begin{array}{l}\overline{\bar{D}} \\
\dot{0} \\
\underline{0} \\
\underline{0}\end{array}$ & $\stackrel{2}{2}$ & $\bar{x}$ \\
\hline & & $\frac{\bar{z}}{0}$ & $\begin{array}{l}\hat{\infty} \\
\infty \\
\dot{ \pm}\end{array}$ & 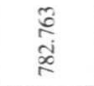 & 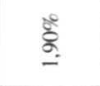 & $\stackrel{\vec{t}}{t}$ \\
\hline & \multirow{5}{*}{ 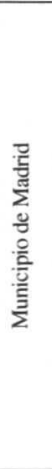 } & 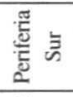 & $\underset{\substack{++}}{+}$ & 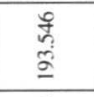 & 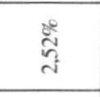 & $\ddot{\alpha}$ \\
\hline & & 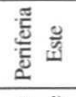 & 号 & $\begin{array}{l}\tilde{A} \\
\tilde{J} \\
\underline{H}\end{array}$ & $\frac{80}{2}$ & $\stackrel{\infty}{\underset{7}{*}}$ \\
\hline & & 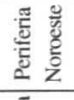 & $\begin{array}{l}\stackrel{a}{\infty} \\
\stackrel{\infty}{=}\end{array}$ & $\begin{array}{l}\frac{\infty}{d} \\
\stackrel{d}{j} \\
\stackrel{f}{f}\end{array}$ & $\stackrel{80}{=}$ & $\stackrel{\vec{\sigma}}{\underline{0}}$ \\
\hline & & 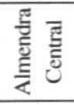 & $\underset{\stackrel{n}{E}}{\stackrel{n}{s}}$ & $\begin{array}{l}\tilde{x} \\
\infty \\
0 \\
\infty\end{array}$ & $\frac{80}{i}$ & $\stackrel{\infty}{\vec{J}}$ \\
\hline & & $\overline{\underline{J}}$ & 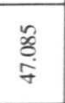 & $\begin{array}{l}0 \\
\frac{0}{8} \\
\frac{8}{8}\end{array}$ & $\begin{array}{l}\theta^{8} \\
\infty \\
i\end{array}$ & $\stackrel{\cong}{\equiv}$ \\
\hline & 傿 & & $\frac{2}{6}$ & 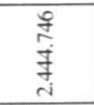 & $\frac{8}{d}$ & $\approx$ \\
\hline ङू & & & $\frac{\circ}{5}$ & $\begin{array}{l}\approx \\
\sigma \\
0 \\
0 \\
ن\end{array}$ & $\underset{n}{*}$ & $\stackrel{8}{8}$ \\
\hline & & & 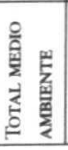 & 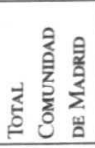 & 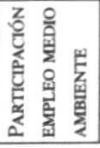 & 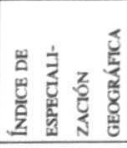 \\
\hline
\end{tabular}

Nota: El índice de especialización geográfica se calcula como el ratio entre el peso del empleo medioambiental en un área y el peso del empleo medioambiental en el conjunto de la Comunidad de Madrid (CM). Por definición el índice para el conjunto de la CM es igual a 100.

S.E.: Fuente: Elaboración propia a partir de datos del Directorio de Unidades de Actividad Económica. Instituto Estadística Comunidad de Madrid. 


\begin{tabular}{|c|c|c|c|c|c|c|c|c|c|c|c|c|c|}
\hline & \multirow{3}{*}{ Total } & \multicolumn{11}{|c|}{ Área Metropolitana } & \multirow{3}{*}{$\begin{array}{c}\text { Municipios } \\
\text { no metro- } \\
\text { politanos }\end{array}$} \\
\hline & & \multirow{2}{*}{ Total } & \multicolumn{5}{|c|}{ Municipio de Madrid } & \multicolumn{5}{|c|}{ Corona Metropolitana } & \\
\hline & & & Total & \begin{tabular}{|c|} 
Almendra \\
Central \\
\end{tabular} & $\begin{array}{l}\text { Periferia } \\
\text { Noroeste }\end{array}$ & \begin{tabular}{|c|} 
Periferia \\
Este
\end{tabular} & \begin{tabular}{|c|} 
Periferia \\
Sur
\end{tabular} & Total & Norte & Este & Sur & Oeste & \\
\hline $\begin{array}{l}\text { Extracción y aglomeración de } \\
\text { antracita, hulla, lignito y turba. }\end{array}$ & 61 & 61 & 61 & 58 & S.E. & 0 & 0 & 0 & 0 & 0 & 0 & 0 & 0 \\
\hline $\begin{array}{l}\text { Extracción de crudos de petróleo } \\
\text { y gas natural. Actividades de } \\
\text { los servicios relacionados con } \\
\text { las explotaciones petrolíferas y } \\
\text { de gas, excepto actividades de } \\
\text { prospección. }\end{array}$ & 579 & 572 & 568 & 461 & S.E. & S.E. & S.E. & S.E. & S.E. & 0 & S.E. & 0 & S.E. \\
\hline $\begin{array}{l}\text { Extracción de minerales de } \\
\text { uranio y torio. }\end{array}$ & S.E. & S.E. & S.E. & 0 & S.E. & 0 & 0 & 0 & 0 & 0 & 0 & 0 & 0 \\
\hline $\begin{array}{l}\text { Extracción de minerales } \\
\text { metálicos. }\end{array}$ & S.E. & S.E. & S.E. & S.E. & 0 & 0 & 0 & 0 & 0 & 0 & 0 & 0 & 0 \\
\hline $\begin{array}{l}\text { Extracción de minerales no } \\
\text { metálicos ni energéticos. }\end{array}$ & 1.346 & 723 & 547 & 322 & 191 & S.E. & 0 & 176 & 0 & 138 & S.E. & S.E. & 623 \\
\hline $\begin{array}{l}\text { Fabricación de maquinaria y } \\
\text { material eléctrico. }\end{array}$ & 241 & 212 & 105 & 23 & 11 & 65 & 7 & 108 & 8 & 54 & 42 & 4 & 29 \\
\hline $\begin{array}{l}\text { Fabricación de equipo } \\
\text { e instrumentos médico- } \\
\text { quirúrgicos, de precisión, óptica } \\
\text { y relojería. }\end{array}$ & 118 & 98 & 43 & 16 & 8 & 10 & 10 & 55 & 14 & 14 & 24 & 3 & 20 \\
\hline $\begin{array}{l}\text { Fabricación de muebles. Otras } \\
\text { industrias manufactureras. }\end{array}$ & 1.683 & 1.153 & 415 & 91 & 89 & 61 & 173 & 738 & 39 & 239 & 436 & 24 & 530 \\
\hline Reciclaje. & 172 & 133 & 60 & 21 & 12 & 20 & 6 & 73 & 1 & 12 & 59 & 1 & 38 \\
\hline
\end{tabular}




\begin{tabular}{|c|c|c|c|c|c|c|c|c|c|c|c|c|c|}
\hline & \multirow{3}{*}{ Total } & \multicolumn{11}{|c|}{ Área Metropolitana } & \multirow{3}{*}{$\begin{array}{c}\text { Municipios } \\
\text { no metro- } \\
\text { politanos }\end{array}$} \\
\hline & & \multirow{2}{*}{ Total } & \multicolumn{5}{|c|}{ Municipio de Madrid } & \multicolumn{5}{|c|}{ Corona Metropolitana } & \\
\hline & & & Total & \begin{tabular}{c|c} 
Almendra \\
Central
\end{tabular} & \begin{tabular}{|l|l} 
Periferia \\
Noroeste
\end{tabular} & $\begin{array}{c}\text { Periferia } \\
\text { Este }\end{array}$ & $\begin{array}{c}\text { Periferia } \\
\text { Sur }\end{array}$ & Total & Norte & Este & Sur & Oeste & \\
\hline $\begin{array}{l}\text { Producción y distribución de } \\
\text { energía eléctrica, gas, vapor y } \\
\text { agua caliente. }\end{array}$ & 11.101 & 10.086 & 8.486 & 1.812 & 4.909 & 807 & 941 & 1.600 & 1.172 & 180 & 124 & 125 & 1.015 \\
\hline $\begin{array}{l}\text { Captación, depuración y } \\
\text { distribución de agua. }\end{array}$ & 3.351 & 2.697 & 2.116 & 1.580 & 421 & S.E. & S.E. & 581 & 158 & 235 & 75 & 113 & 654 \\
\hline Hostelería. & 12.225 & 11.318 & 8.389 & 4.639 & 2.419 & 429 & 831 & 2.929 & 528 & 573 & 1.169 & 659 & 907 \\
\hline $\begin{array}{l}\text { Transporte terrestre. Transporte } \\
\text { por tubería. }\end{array}$ & 5.150 & 4.646 & 2.866 & 1.584 & 481 & 282 & 479 & 1.781 & 114 & 742 & 817 & 109 & 504 \\
\hline $\begin{array}{l}\text { Transporte marítimo y por vías } \\
\text { de navegación interiores }\end{array}$ & 146 & 146 & 100 & 97 & 0 & S.E. & 0 & 47 & 43 & 0 & S.E. & 0 & 0 \\
\hline Transporte aéreo y espacial. & 6.061 & 6.058 & 5.983 & 496 & 5.367 & 103 & S.E. & 75 & 13 & 38 & S.E. & S.E. & S.E. \\
\hline $\begin{array}{l}\text { Actividades anexas a los } \\
\text { transportes; actividades de } \\
\text { agencias de viajes. }\end{array}$ & 2.742 & 2.608 & 1.886 & 917 & 738 & 147 & 76 & 722 & 119 & 349 & 125 & 129 & 134 \\
\hline Otras actividades empresariales. & 1.544 & 1.500 & 1.158 & 678 & 269 & 133 & 75 & 341 & 117 & 61 & 90 & 73 & 44 \\
\hline \begin{tabular}{|l|} 
Administración pública, defensa \\
y seguridad social obligatoria.
\end{tabular} & 1.405 & 1.290 & 1.102 & 822 & 187 & 15 & 72 & 188 & 17 & 55 & 79 & 37 & 115 \\
\hline $\begin{array}{l}\text { Actividades de saneamiento } \\
\text { público. }\end{array}$ & 2.674 & 2.477 & 1.583 & 228 & 310 & 353 & 683 & 895 & 64 & 240 & 470 & 121 & 197 \\
\hline Actividades asociativas. & 3.006 & 2.844 & 2.498 & 1.812 & 482 & 77 & 115 & 346 & 103 & 82 & 105 & 57 & 162 \\
\hline
\end{tabular}




\begin{tabular}{|c|c|c|c|c|c|c|c|c|c|c|c|c|c|}
\hline & \multirow{3}{*}{ Total } & \multicolumn{11}{|c|}{ Área Metropolitana } & \multirow{3}{*}{$\begin{array}{c}\text { Municipios } \\
\text { no metro- } \\
\text { politanos }\end{array}$} \\
\hline & & \multirow{2}{*}{ Total } & \multicolumn{5}{|c|}{ Municipio de Madrid } & \multicolumn{5}{|c|}{ Corona Metropolitana } & \\
\hline & & & Total & $\begin{array}{c}\text { Almendra } \\
\text { Central }\end{array}$ & \begin{tabular}{|c|} 
Periferia \\
Noroeste
\end{tabular} & $\begin{array}{c}\text { Periferia } \\
\text { Este }\end{array}$ & $\begin{array}{c}\text { Periferia } \\
\text { Sur }\end{array}$ & Total & Norte & Este & Sur & Oeste & \\
\hline $\begin{array}{l}\text { Actividades recreativas, } \\
\text { culturales y deportivas. }\end{array}$ & 10.583 & 9.898 & 6.824 & 3.885 & 1.393 & 290 & 1.021 & 3.075 & 690 & 357 & 537 & 1.491 & 685 \\
\hline $\begin{array}{l}\text { Actividades diversas de } \\
\text { servicios personales. }\end{array}$ & 3.788 & 3.454 & 2.296 & 1.235 & 503 & 147 & 386 & 1.158 & 156 & 278 & 516 & 208 & 334 \\
\hline TOTAL MEDIO AMBIENTE & 67.976 & 61.976 & 47.085 & 20.775 & 17.789 & 2.940 & 4.874 & 14.887 & 3.356 & 3.646 & 4.668 & 3.151 & 5.990 \\
\hline $\begin{array}{l}\text { TOTAL COMUNIDAD DE } \\
\text { MADRID }\end{array}$ & 2.668 .935 & 2.444 .746 & 1.661 .983 & 860.877 & 432.748 & 154.222 & 193.546 & 782.763 & 168.811 & 183.213 & 285.073 & 145.666 & 224.189 \\
\hline$\%$ Medio ambiente & $2,5 \%$ & $2,5 \%$ & $2,8 \%$ & $2,4 \%$ & $4,1 \%$ & $1,9 \%$ & $2,5 \%$ & $1,9 \%$ & $2,0 \%$ & $2,0 \%$ & $1,6 \%$ & $2,2 \%$ & $2,7 \%$ \\
\hline
\end{tabular}




\begin{tabular}{|c|c|c|c|c|c|c|c|c|c|c|c|c|c|}
\hline & \multicolumn{13}{|c|}{ Zona Metropolitana de Residencia } \\
\hline & \multirow{3}{*}{ Total } & \multicolumn{11}{|c|}{ Área Metropolitana } & \multirow{3}{*}{$\begin{array}{c}\text { Municipios } \\
\text { no metro- } \\
\text { politanos }\end{array}$} \\
\hline & & \multirow[b]{2}{*}{ Total } & \multicolumn{5}{|c|}{ Municipio de Madrid } & \multicolumn{5}{|c|}{ Corona Metropolitana } & \\
\hline & & & Total & $\begin{array}{l}\text { Almendra } \\
\text { Central }\end{array}$ & $\begin{array}{l}\text { Periferia } \\
\text { Noroeste }\end{array}$ & $\begin{array}{l}\text { Periferia } \\
\text { Este }\end{array}$ & $\begin{array}{c}\text { Periferia } \\
\text { Sur }\end{array}$ & Total & Norte & Este & Sur & Oeste & \\
\hline $\begin{array}{l}\text { Extracción y aglomeración de } \\
\text { antracita, hulla, lignito y turba. }\end{array}$ & 100 & 109 & 161 & 295 & S.E. & 0 & 0 & 0 & 0 & 0 & 0 & 0 & 0 \\
\hline $\begin{array}{l}\text { Extracción de crudos de petróleo } \\
\text { y gas natural. Actividades de } \\
\text { los servicios relacionados con } \\
\text { las explotaciones petroliferas y } \\
\text { de gas, excepto actividades de } \\
\text { prospección. }\end{array}$ & 100 & 108 & 158 & 247 & S.E. & S.E. & S.E. & S.E. & S.E. & 0 & S.E. & 0 & S.E. \\
\hline $\begin{array}{l}\text { Extracción de minerales de uranio } \\
\text { y torio. }\end{array}$ & S.E. & S.E. & S.E. & S.E. & S.E. & S.E. & S.E. & S.E. & S.E. & S.E. & S.E. & S.E. & S.E. \\
\hline $\begin{array}{l}\text { Extracción de minerales } \\
\text { metálicos. }\end{array}$ & S.E. & S.E. & S.E. & S.E. & S.E. & S.E. & S.E. & S.E. & S.E. & S.E. & S.E. & S.E. & S.E. \\
\hline $\begin{array}{l}\text { Extracción de minerales no } \\
\text { metálicos ni energéticos. }\end{array}$ & 100 & 59 & 65 & 74 & 88 & S.E. & 0 & 45 & 0 & 149 & S.E. & S.E. & 551 \\
\hline $\begin{array}{l}\text { Fabricación de maquinaria y } \\
\text { material eléctrico. }\end{array}$ & 100 & 96 & 70 & 29 & 27 & 468 & 37 & 152 & 54 & 324 & 162 & 28 & 142 \\
\hline $\begin{array}{l}\text { Fabricación de equipo e } \\
\text { instrumentos médico-quirúrgicos, } \\
\text { de precisión, óptica y relojería. }\end{array}$ & 100 & 91 & 59 & 41 & 42 & 142 & 119 & 158 & 182 & 175 & 191 & 44 & 202 \\
\hline $\begin{array}{l}\text { Fabricación de muebles. Otras } \\
\text { industrias manufactureras. }\end{array}$ & 100 & 75 & 40 & 17 & 32 & 63 & 142 & 150 & 36 & 207 & 243 & 26 & 375 \\
\hline Reciclaje. & 100 & 85 & 56 & 37 & 45 & 206 & 51 & 146 & 11 & 103 & 322 & 11 & 266 \\
\hline $\begin{array}{l}\text { Producción y distribución de } \\
\text { energía eléctrica, gas, vapor y } \\
\text { agua caliente. }\end{array}$ & 100 & 99 & 123 & 51 & 273 & 126 & 117 & 49 & 167 & 24 & 10 & 21 & 109 \\
\hline
\end{tabular}




\begin{tabular}{|c|c|c|c|c|c|c|c|c|c|c|c|c|c|}
\hline & \multicolumn{13}{|c|}{ Zona Metropolitana de Residencia } \\
\hline & \multirow{3}{*}{ Total } & \multicolumn{11}{|c|}{ Área Metropolitana } & \multirow{3}{*}{$\begin{array}{c}\text { Municipios } \\
\text { no metro- } \\
\text { politanos }\end{array}$} \\
\hline & & \multirow[b]{2}{*}{ Total } & \multicolumn{5}{|c|}{ Municipio de Madrid } & \multicolumn{5}{|c|}{ Corona Metropolitana } & \\
\hline & & & Total & $\begin{array}{c}\text { Almendra } \\
\text { Central } \\
\end{array}$ & $\begin{array}{c}\text { Periferia } \\
\text { Noroeste }\end{array}$ & $\begin{array}{c}\text { Periferia } \\
\text { Este }\end{array}$ & $\begin{array}{c}\text { Periferia } \\
\text { Sur }\end{array}$ & Total & Norte & Este & Sur & Oeste & \\
\hline $\begin{array}{l}\text { Captación, depuración y distribución } \\
\text { de agua. }\end{array}$ & 100 & 88 & 101 & 146 & 77 & S.E. & S.E. & 59 & 75 & 102 & 21 & 62 & 232 \\
\hline Hostelería. & 100 & 101 & 110 & 118 & 122 & 61 & 94 & 82 & 68 & 68 & 90 & 99 & 88 \\
\hline $\begin{array}{l}\text { Transporte terrestre. Transporte por } \\
\text { tubería. }\end{array}$ & 100 & 98 & 89 & 95 & 58 & 95 & 128 & 118 & 35 & 210 & 149 & 39 & 116 \\
\hline $\begin{array}{l}\text { Transporte marítimo y por vías de } \\
\text { navegación interiores }\end{array}$ & 100 & 109 & 109 & 205 & 0 & S.E. & 0 & 109 & 468 & 0 & S.E. & 0 & 0 \\
\hline Transporte aéreo y espacial. & 100 & 109 & 159 & 25 & 546 & 29 & S.E. & 4 & 3 & 9 & S.E. & S.E. & S.E. \\
\hline $\begin{array}{l}\text { Actividades anexas a los transportes; } \\
\text { actividades de agencias de viajes. }\end{array}$ & 100 & 104 & 110 & 104 & 166 & 93 & 38 & 90 & 69 & 185 & 43 & 86 & 58 \\
\hline Otras actividades empresariales. & 100 & 106 & 120 & 136 & 107 & 149 & 67 & 75 & 120 & 58 & 55 & 86 & 34 \\
\hline $\begin{array}{l}\text { Administración pública, defensa y } \\
\text { seguridad social obligatoria. }\end{array}$ & 100 & 100 & 126 & 181 & 82 & 18 & 71 & 46 & 19 & 57 & 53 & 48 & 98 \\
\hline Actividades de saneamiento público. & 100 & 101 & 95 & 26 & 71 & 229 & 352 & 114 & 38 & 131 & 164 & 83 & 88 \\
\hline Actividades asociativas. & 100 & 103 & 133 & 187 & 99 & 45 & 53 & 39 & 54 & 40 & 33 & 34 & 64 \\
\hline $\begin{array}{l}\text { Actividades recreativas, culturales y } \\
\text { deportivas. }\end{array}$ & 100 & 102 & 104 & 114 & 81 & 47 & 133 & 99 & 103 & 49 & 47 & 258 & 77 \\
\hline $\begin{array}{l}\text { Actividades diversas de servicios } \\
\text { personales. }\end{array}$ & 100 & 100 & 97 & 101 & 82 & 67 & 140 & 104 & 65 & 107 & 128 & 101 & 105 \\
\hline TOTAL MEDIO AMBIENTE & 100 & 100 & 111 & 95 & 161 & 75 & 99 & 75 & 78 & 78 & 64 & 85 & 105 \\
\hline TOTAL COMUNIDAD DE MADRID & 100 & 100 & 100 & 100 & 100 & 100 & 100 & 100 & 100 & 100 & 100 & 100 & 100 \\
\hline
\end{tabular}


Los índices de especialización calculados por grandes área geográficas muestran una mayor especialización en el Municipio de Madrid, especialmente en la periferia Noroeste, y en los municipios no metropolitanos. En el resto de casos las actividades medioambientales presentan una concentración inferior a la del empleo total. Este caso es particularmente significativo en la corona metropolitana sur, Periferia Este del municipio de Madrid y la corona metropolitana norte y este.

Figura 7.-Índice de especialización geográfica del empleo. $2007^{14}$

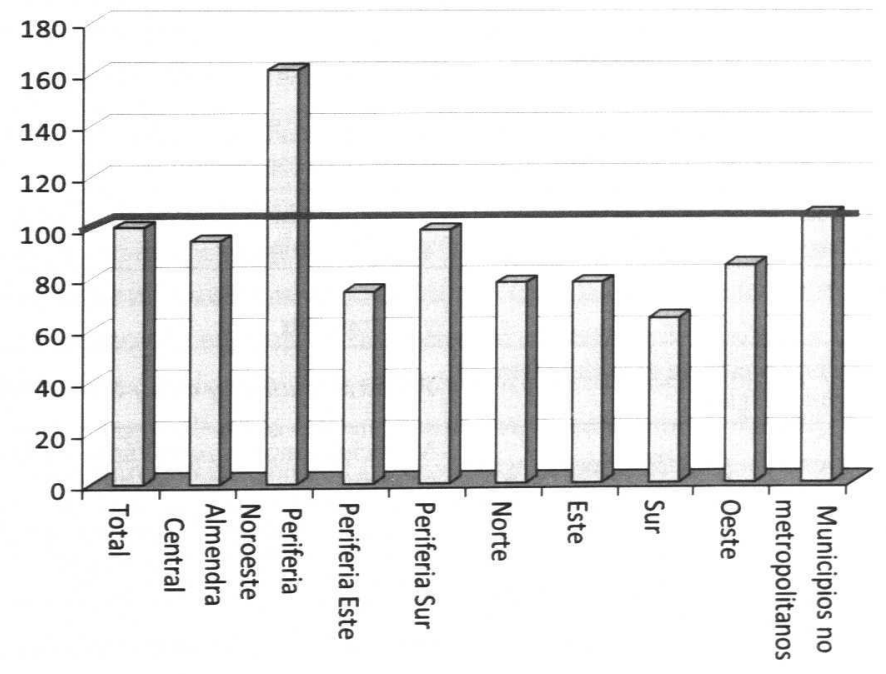

Fuente: Elaboración propia a partir de datos del Directorio de Unidades de Actividad Económica. Instituto Estadística Comunidad de Madrid.

En la tabla siguiente se muestra con mayor detalle las áreas geográficas en las que los distintos sectores medioambientales presentan una mayor concentración en términos relativos.

14 Nota: El índice de especialización geográfica se calcula como el ratio entre el peso del empleo medioambiental en un área y el peso del empleo medioambiental en el conjunto de la Comunidad de Madrid (CM). Por definición el índice para el conjunto de la CM es igual a 100. 
TABLA 13.-Actividades vinculadas al medioambiente y especialización geográfica

\begin{tabular}{|c|c|}
\hline Sector & Área geográfica de mayor especialización \\
\hline $\begin{array}{l}\text { Extracción y aglomeración de antracita, hulla, } \\
\text { lignito y turba. }\end{array}$ & Almendra Central \\
\hline $\begin{array}{l}\text { Extracción de crudos de petróleo y gas natural. } \\
\text { Actividades de los servicios relacionados con } \\
\text { las explotaciones petrolíferas y de gas, excepto } \\
\text { actividades de prospección. }\end{array}$ & Almendra Central \\
\hline $\begin{array}{l}\text { Extracción de minerales no metálicos ni } \\
\text { energéticos. }\end{array}$ & Municipios no metropolitanos y Corona norte \\
\hline $\begin{array}{l}\text { Fabricación de maquinaria y material } \\
\text { eléctrico. }\end{array}$ & Periferia Este y Corona Este y Sur \\
\hline $\begin{array}{l}\text { Fabricación de equipo e instrumentos médico- } \\
\text { quirúrgicos, de precisión, óptica y relojería. }\end{array}$ & $\begin{array}{l}\text { Municipios no metropolitanos, Corona norte y } \\
\text { sur y Periferia Este }\end{array}$ \\
\hline $\begin{array}{l}\text { Fabricación de muebles. Otras industrias } \\
\text { manufactureras. }\end{array}$ & $\begin{array}{l}\text { Municipios no metropolitanos, Corona este y } \\
\text { sur y Periferia sur }\end{array}$ \\
\hline Reciclaje. & $\begin{array}{l}\text { Municipios no metropolitanos, Periferia Este } \\
\text { y Corona Sur }\end{array}$ \\
\hline $\begin{array}{l}\text { Producción y distribución de energía eléctrica, } \\
\text { gas, vapor y agua caliente. }\end{array}$ & Periferia noroeste y este y corona norte \\
\hline Captación, depuración y distribución de agua. & $\begin{array}{l}\text { Municipios no metropolitanos y Almendra } \\
\text { central }\end{array}$ \\
\hline Hostelería. & Periferia Noroeste y Almendra central \\
\hline Transporte terrestre. Transporte por tubería. & Corona Este, Sur y Periferia Sur \\
\hline $\begin{array}{l}\text { Transporte marítimo y por vías de navegación } \\
\text { interiores }\end{array}$ & Corona Norte y Almendra central \\
\hline Transporte aéreo y espacial. & Periferia Noroeste \\
\hline $\begin{array}{l}\text { Actividades anexas a los transportes; } \\
\text { actividades de agencias de viajes. }\end{array}$ & Periferia Noroeste y Corona Este \\
\hline Otras actividades empresariales. & Periferia Este y corona norte \\
\hline $\begin{array}{l}\text { Administración pública, defensa y seguridad } \\
\text { social obligatoria. }\end{array}$ & Almendra central \\
\hline Actividades de saneamiento público. & Periferia Este y Sur y Corona Sur y Este \\
\hline Actividades asociativas. & Almendra Central \\
\hline $\begin{array}{l}\text { Actividades recreativas, culturales y } \\
\text { deportivas. }\end{array}$ & Corona Oeste y Periferia Sur \\
\hline Actividades diversas de servicios personales. & Periferia Sur y Corona Sur \\
\hline
\end{tabular}

Fuente: Elaboración propia. 


\section{Conclusiones}

Para la elaboración del presente artículo, tras analizar diferentes delimitaciones conceptuales del sector medioambiental, se ha determinado seguir la definición establecida en el trabajo «Links between the enviroment, economy and jobs» elaborado por GHK en colaboración con Cambridge Econometrics y el Institute for European Environmental Policy encargado por la Dirección General de Medio Ambiente de la Comisión Europea. A pesar de las limitaciones de este enfoque, tiene la ventaja de permitir obtener estimaciones que de otra manera sería inabordables. En base a dicha definición se ha estimado el valor en términos de empleo del sector medio ambiental en la Comunidad de Madrid considerando diferentes fuentes estadísticas.

De acuerdo con las estimaciones realizadas, el peso del sector medioambiental, en materia de empleo, sobre el total de la economía de la Comunidad de Madrid, se sitúa entre el 3\% y el 4\%. Si bien no parece una cifra muy elevada, lo importante es el potencial de crecimiento de dicho sector. Así, la Comunidad de Madrid ha experimentado un importante avance en los últimos años, con una tasa de crecimiento acumulado de más del 40\% en términos de número de ocupados en el período 2001-2007.

Asimismo de acuerdo con estas estimaciones, dentro del conjunto de actividades económicas es la agricultura la que supone el mayor peso dentro del empleo medioambiental de la Comunidad de Madrid, llegando a superar el 30\% en algunos años. Son la hostelería así como la producción y distribución de energía eléctrica las otras dos ramas con mayor importancia en estos términos.

Los datos sobre los puestos de trabajo anexos al sector medioambiental confirman la importancia del mismo en términos del empleo, un 3,8\% del total de empleo para el año 2006; y validan el importante crecimiento acaecido dentro de la Comunidad de Madrid en los últimos años, el 16\% desde el año 2000 hasta el 2006.

Las actividades medioambientales presentan una distribución geográfica del empleo similar al del conjunto de actividades económicas de la Comunidad de Madrid. Esta distribución se caracteriza por una notable concentración del empleo en el municipio de Madrid, dónde se concentraría el 69,3\% del empleo del sector, y la corona metropolitana, que supone el $21,9 \%$ del empleo medioambiental.

Comparando la estructura del empleo del total de la economía regional y del sector medioambiental puede apreciarse en este último caso una tendencia a la concentración en el municipio de Madrid, especialmente en la periferia Noroeste (Fuencarral-El Pardo, Moncloa-Aravaca, Ciudad Lineal, Hortaleza, Barajas), y en el área no metropolitana.

Dadas las limitaciones de la información secundaria empleada en las estimaciones, una de las posibles líneas de investigación futura sería la estimación mediante encuesta 
de los pesos asignados a las actividades medioambientales para cada una de las ramas CNAE en la Comunidad de Madrid, lo cual permitiría desagregar las estimaciones en cada una de los tipos de actividades que se incluyen en el sector medioambiental (aquellas en las que el medio ambiente es un recurso primario, las relacionadas con la protección y la gestión del medio ambiente y las actividades dependientes de la calidad del medio ambiente).

\section{Bibliografía}

Commission Of The European Communities (2004), The effects of environmental policy on European business and its competitiveness - a framework for analysis. Commission Staff Working Paper. SEC (2004) núm. 769, de 10 de junio de 2004.

- (2005), On the links between employment policies and environment policies. Commission Staff Working Paper. SEC (2005) 1530, de 17 de noviembre de 2005.

Comunidad de MAdrid (2006), Panorama laboral 2006: informe sobre el sector de las tecnologías medioambientales en la Comunidad de Madrid. Consejería de Empleo, Madrid.

ECORIS (2008), Environment and labour force skills. Para la D.G. Environment of the Commission of the European Communities.

ECOTEC (2002), Analysis of the EU eco-industries, their employment and export potential. Para la D.G. Environment of the Commission of the European Communities.

ERNEST \& Young (2006), Eco-industry, its size, employment, perspectives and barriers to growth in an enlarged EU. Para la D.G. Environment of the Commission of the European Communities.

EUROSTAT (1994), Sistema Europeo para la Recogida de Información Económica sobre el Medio Ambiente (European System for the Collection of Economic Information on the Environment). Manual Serie E, EUROSTAT E8. Serie E, Bruselas.

- (1996), Ficheros de empresas para fines estadísticos: recomendaciones metodológicas. Vol. 1, Luxemburgo.

- (2000), Clasificación de actividades y gastos de protección del medio ambiente.

- AND DG XI DE LA Comisión Europea (1994), An estimate of eco-industries in the European Union. Summary Report.

Fundación Entorno, Empresa y Medio Ambiente (2000), Empleo y formación en el sector del medio ambiente en España, Madrid.

GHK (2007), Links between the environment, economy and jobs. Para la D.G. Environment of the Commission of the European Communities. 
Legislación eSPAÑola, Ley 16/2002, de 1 de julio, de prevención y control integrados de la contaminación, BOE núm. 157 02/07/2002.

OCDE (1995), The environment industry and markets in selected Central and Eastern European countries, 1995, Paris.

- (1997), Sustainable growth and employment: empirical analyses, París.

- (2007), Issues of dual use and reviewing product coverage of environmental goods. OCDE Trade and Environment Working Paper núm. 2007-01, COM/ENV/TD (2006)30 FINAL, París.

- y EUROSTAT (1999), The environment goods and services industry: manual for data collection and analysis, París.

- y EUROSTAT (2005), The environmental protection expenditure and revenue. Joint questionnaire/SERIEE environmental protection expenditure account, París.

Price Waterhouse Coopers (2000), Estimación del empleo ambiental en España, Ministerio de Medio Ambiente, Madrid. 


\section{ANEXO 1. ZONAS METROPOLITANAS.}

FIGURA A.I.—Áreas geográficas Comunidad de Madrid

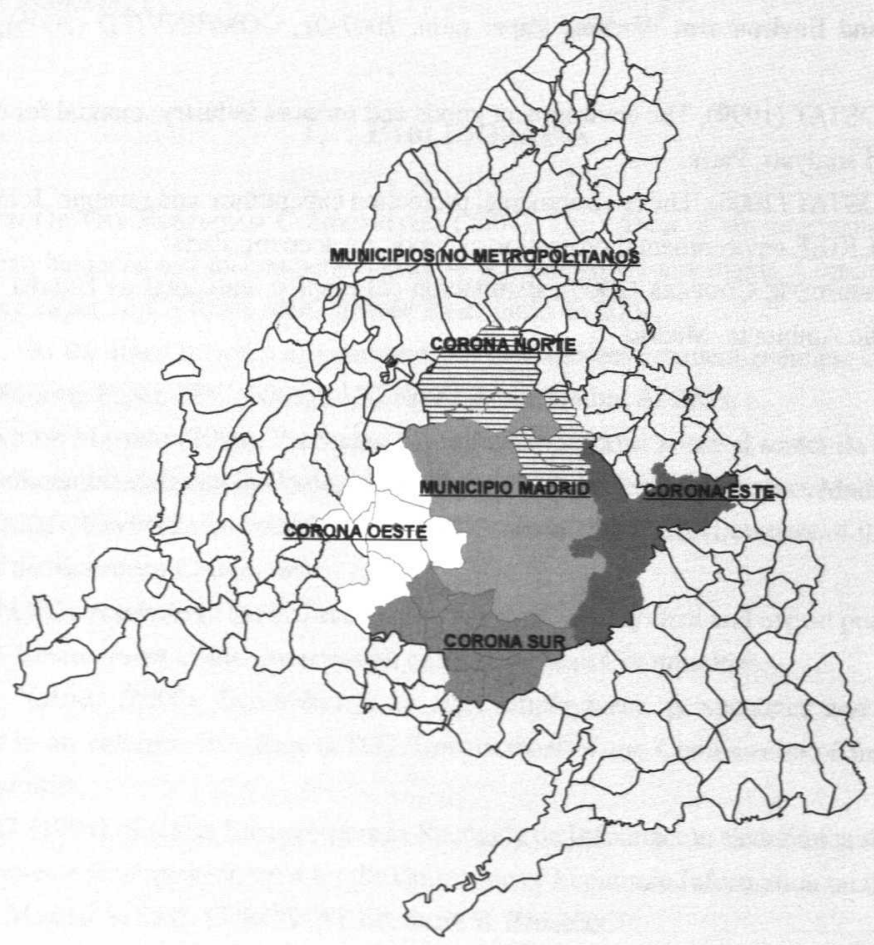




\begin{tabular}{|c|c|}
\hline \multicolumn{2}{|c|}{ Municipios que configuran el Área Metropolitana } \\
\hline \multicolumn{2}{|c|}{ MADRID: } \\
\hline $\begin{array}{l}\text { ALMENDRA CENTRAL: } \\
\text { DISTRITOS: } \\
\text { Centro } \\
\text { Arganzuela } \\
\text { Retiro } \\
\text { Salamanca } \\
\text { Chamartín } \\
\text { Tetuán } \\
\text { Chamberí } \\
\end{array}$ & $\begin{array}{l}\text { PERIFERIA ESTE: } \\
\text { DISTRITOS: } \\
\text { Moratalaz } \\
\text { Villa de Vallecas } \\
\text { Vicálvaro } \\
\text { San Blas }\end{array}$ \\
\hline $\begin{array}{l}\text { PERIFERIA NOROESTE: } \\
\text { DISTRITOS: } \\
\text { Fuencarral-El Pardo } \\
\text { Moncloa-Aravaca } \\
\text { Ciudad Lineal } \\
\text { Hortaleza } \\
\text { Barajas }\end{array}$ & $\begin{array}{l}\text { PERIFERIA SUR: } \\
\text { DISTRITOS: } \\
\text { Latina } \\
\text { Carabanchel } \\
\text { Usera } \\
\text { Puente de Vallecas } \\
\text { Villaverde }\end{array}$ \\
\hline \multicolumn{2}{|c|}{ CORONA METROPOLITANA: } \\
\hline $\begin{array}{ll}\text { NORTE: } & \\
& \text { Alcobendas } \\
& \text { Colmenar Viejo } \\
& \text { San Sebastián de los } \\
& \text { Reyes } \\
& \text { Tres Cantos }\end{array}$ & $\begin{array}{l}\text { Alcorcón } \\
\text { Fuenlabrada } \\
\text { Getafe } \\
\text { Leganés } \\
\text { Móstoles } \\
\text { Parla } \\
\text { Pinto }\end{array}$ \\
\hline $\begin{array}{ll}\text { ESTE: } & \\
& \text { Alcalá de Henares } \\
& \text { Coslada } \\
& \text { Mejorada del Campo } \\
& \text { Paracuellos de Jarama } \\
& \text { Rivas-Vaciamadrid } \\
& \text { San Fernando de Henares } \\
& \text { Torrejón de Ardóz } \\
& \text { Velilla de San Antonio }\end{array}$ & $\begin{array}{l}\text { OESTE: } \\
\text { Boadilla del Monte } \\
\text { Majadahonda } \\
\text { Pozuelo de Alarcón } \\
\text { Rozas de Madrid (Las) } \\
\text { Villanueva de la Cañada } \\
\text { Villanueva del Pardillo } \\
\text { Villaviciosa de Odón }\end{array}$ \\
\hline \multicolumn{2}{|c|}{$\begin{array}{l}\text { MUNICIPIOS NO METROPOLITANOS: } \\
\text { Resto de municipios. }\end{array}$} \\
\hline
\end{tabular}

